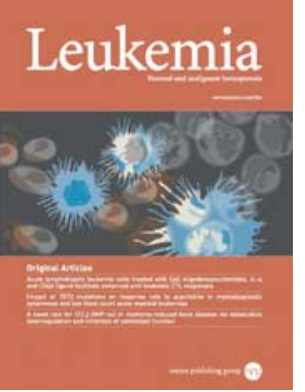

Bone marrow fibroblasts parallel multiple myeloma progression in patients and mice: in vitro and in vivo studies

M A Frassanito, L Rao, M Moschetta, R Ria, L Di Marzo, A De Luisi, V Racanelli, I Catacchio, S Berardi, A Basile, E Menu, S Ruggieri, B Nico, D Ribatti, R Fumarulo, F Dammacco, K Vanderkerken, A Vacca

Cite this article as: $M$ A Frassanito, L Rao, M Moschetta, R Ria, L Di Marzo, A De Luisi, V Racanelli, I Catacchio, S Berardi, A Basile, E Menu, S Ruggieri, B Nico, D Ribatti, R Fumarulo, F Dammacco, K Vanderkerken, A Vacca, Bone marrow fibroblasts parallel multiple myeloma progression in patients and mice: in vitro and in vivo studies, Leukemia accepted article preview 2 September 2013; doi: $10.1038 /$ leu.2013.254.

This is a PDF file of an unedited peer-reviewed manuscript that has been accepted for publication. NPG are providing this early version of the manuscript as a service to our customers. The manuscript will undergo copyediting, typesetting and a proof review before it is published in its final form. Please note that during the production process errors may be discovered which could affect the content, and all legal disclaimers apply.

Received 10 July 2013; accepted 22 August 2013; Accepted article preview online 2 September 2013 


\section{BONE MARROW FIBROBLASTS PARALEEL MULTIPLE MYELOMA \\ PROGRESSION IN PATIENTS AND MICE: IN VITRO AND IN VIVO STUDIES}

Maria Antonia Frassanito ${ }^{1}$, Luigia Rao ${ }^{2}$, Michele Moschetta ${ }^{2}$, Roberto Ria ${ }^{2}$, Lucia Di Marzo ${ }^{2}$, Annunziata De Luisi², Vito Racanelli ${ }^{2}$, Ivana Catacchio ${ }^{2}$, Simona Berardi ${ }^{2}$, Antonio Basile ${ }^{2}$, Eline Menu ${ }^{3}$, Simona Ruggieri ${ }^{4}$, Beatrice $\mathrm{Nico}^{4}$, Domenico Ribatti ${ }^{4}$, Ruggiero Fumarulo ${ }^{1}$, Franco Dammacco ${ }^{2}$, Karin Vanderkerken ${ }^{3}$, Angelo Vacca ${ }^{2}$

${ }^{1}$ Department of Biomedical Sciences and Human Oncology, General Pathology Section and ${ }^{2}$ Internal Medicine Section, University of Bari Medical School, Bari, Italy; ${ }^{3}$ Department of Hematology and Immunology, Myeloma Center Brussels, Vrije Universiteit Brussel, Brussels, Belgium; ${ }^{4}$ Department of Human Anatomy, Histology and Embryology, University of Bari Medical School, Bari, Italy.

Running title: Fibroblasts in MM

Correspondence:

Co-correspondence:

1 Prof. Angelo Vacca, M.D.

2 Department of Biomedical Sciences and

3 Human Oncology (DIMO),

4 Section of Internal Medicine and

5 Clinical Oncology,

6 Policlinico - Piazza Giulio Cesare, 11,

7 I-70124 Bari (BA, Italy)

8 Phone: +39-080-5478895

9 Fax: +39-080-559.21.89

10 E-mail: angelo.vacca@uniba.it
11 Prof. Karin Vanderkerken, Ph.D.

12 Department of Hematology and

13 Immunology, Myeloma Center,

14 Laarbeerklaan 103,

15 Vrije Universiteit Brussel (VUB)

16 B-1090 Brussels (Belgium)

17 Phone: +32-2-4774418

18 Fax: +32-2-4774405

19 E-mail: karin.vanderkerken@vub.ac.be

Keywords: Cancer-associated fibroblasts, multiple myeloma, tumor microenvironment 


\section{ACCEPTEDABSTRACT PREVIEW}

The role of cancer-associated fibroblasts (CAFs) has not been previously studied in multiple myeloma (MM). Here, cytofluorimetric analysis revealed higher proportions of bone marrow (BM) CAFs in patients with active MM (both at diagnosis and relapse) compared to patients in remission or those with monoclonal gammopathy of undetermined significance (MGUS) or deficiency anemia (controls). CAFs from MM patients produced increased levels of TGF $\beta$, IL-6, SDF1 $\alpha$, IGF1, VEGF, and FGF2 and displayed an activated and heterogeneous phenotype, which supported their origin from resident fibroblasts, endothelial cells (ECs), and hematopoietic stem and progenitor cells (HSPCs) via the endothelial-mesenchymal transition (EndMT) as well as mesenchymal stem cells via the mesenchymal transition (MT), as both of these processes are induced by MM cells and CAFs. Active MM CAFs fostered chemotaxis, adhesion, proliferation, and apoptosis resistance in MM cells through cytokine signals and cell-to-cell contact, that were inhibited by blocking CXCR4, several integrins, and fibronectin (FN). MM cells also induced the CAFs proliferation. In syngeneic 5T33MM and xenograft mouse models, MM cells induced the expansion of CAFs, which, in turn, promoted MM initiation and progression as well as angiogenesis. In BM biopsies from patients and mice, nests of CAFs were found in close contact with MM cells, suggesting a supportive niche. Therefore, the targeting of CAFs in MM patients may be envisaged as a novel therapeutic strategy. 


\section{ACCEPTINTRODUUCTIONREVIEW}

Tumor growth results from complex interactions between stromal cells and tumor cells, in which the former cell type regulates tumor development, progression, and drug resistance ${ }^{1,2}$. The stromal microenvironment is characterized by a modified extracellular matrix (ECM), enhanced angiogenesis, and cells with an activated phenotype, including fibroblasts referred to as "activated myofibroblasts" or "cancer-associated fibroblasts" (CAFs) ${ }^{2}$. In breast, prostate, and pancreatic carcinomas, the number of CAFs is associated with an increased malignancy grade, tumor progression, and poor prognosis ${ }^{3-6}$. Similarly, in mouse xenografts, CAFs inoculated with carcinoma cells promote tumor survival, proliferation, and invasive behavior $^{7,8}$.

CAFs are heterogeneous ${ }^{9-11}$ and display phenotypes similar to those of myofibroblasts derived from quiescent fibroblasts that have undergone activation during tissue remodeling in wound healing, fibrosis. CAFs express $\alpha$-smooth muscle actin ( $\alpha \mathrm{SMA})$, fibroblast-specific protein-1 (FSP1), fibroblast activation protein (FAP), and the platelet-derived growth factor receptor $\beta$ (PDGFR $\beta$ ); in addition, FAP and $\alpha$ SMA are highly expressed by myofibroblasts ${ }^{10}$, and FSP1 is expressed by both resting fibroblasts and myofibroblasts ${ }^{11}$. The diverse heterogeneity of CAFs likely results from their different origins ${ }^{12-17}$, as CAFs can arise from resident fibroblasts, bone marrow (BM)-derived progenitor cells ${ }^{14,15}$, and cells undergoing the endothelial-mesenchymal transition $(\text { EndMT })^{16}$ or mesenchymal transition $(\mathrm{MT})^{17}$. However, the molecular mechanisms by which fibroblasts are recruited to the tumor and undergo subsequent induction by the microenvironment to become mature and activated fibroblasts remain unclear. Mechanical tension is potentially required for the acquisition and maintenance of the myofibroblast phenotype ${ }^{18}$, as fibroblasts acquire contractile fibers ("microfilament bundles") and express $\alpha$ SMA in response to mechanical stress. During this process, the conversion of physical stimulation into chemical signals ("mechanotransduction") leads to the activation of cancer-promoting signals ${ }^{19,20}$. In addition, 
previous work by Kojma $\hat{e} t a l{ }^{2}$ demonstrated that transforming growth factor- $\beta$ (TGF $\beta$ ) and stromal cell derived factor- $1 \alpha(\mathrm{SDF} 1 \alpha)$ act in autocrine loops to initiate and maintain fibroblast differentiation into myofibroblasts.

In multiple myeloma (MM), plasma cells home to and expand in the BM by establishing pathophysiological interactions with BM stromal cells (BMSCs) and the ECM, which promote their survival, proliferation, and resistance to drugs ${ }^{22}$. The existence and the role of CAFs in MM have not previously been investigated. Therefore, we analyzed the phenotype and function of $\mathrm{BM}$ CAFs in patients with $\mathrm{MM}$ and monoclonal gammopathies of undetermined significance (MGUS). The reciprocal effect of CAFs on MM cell function was studied both in vitro and in vivo using syngeneic 5T33MM and MM xenograft mouse models.

\section{PATIENTS AND METHODS}

\section{Patients}

Fifty-six patients fulfilling the International Myeloma Working Group diagnostic criteria for MM ( $\mathrm{n}=38)$ and MGUS $(\mathrm{n}=18)$ were studied. Twenty-three MM patients had active disease ('active MM'); of these, 14 patients were newly diagnosed and symptomatic, and 9 patients suffered from relapsed MM. Of the patients with active MM, 13 were male, and 10 were female. The age range of this group was 45-88 years with a median of 64.5 years, and the patients were classified according to the Durie \& Salmon (D\&S) stage as IIB (n=2), IIIA $(n=16)$, or IIIB $(n=5)$. The remaining 15 patients had complete or partial remission ('nonactive MM'); of these, 9 were male, and 6 were female. These patients ranged from 42 to 81 years of age, with a median age of 62.3 years. Eleven of the MGUS patients were male, and 7 were female. These MGUS patients were between the ages of 38 and 85 years, with a median age of 62.5 years. Eleven patients with anemia due to iron or vitamin $\mathrm{B}_{12}$ deficiency were studied as controls ${ }^{23}$. Of the control patients, 7 were male, and 4 were female. These patients' ages ranged from 40 to 84 years, with a median of 63.4 years. The study was 
approved by the University of Bari Ethics Committee, and alF patients provided informed consent according to the Declaration of Helsinki.

\section{Cell cultures}

BM mononuclear cells (BMMCs) were isolated by Ficoll-Hypaque gradient separation from heparinized samples and processed with the appropriate separation procedure according to the cell type, as detailed in the Supplementary Methods. The cell purity (>95\%) of all cell populations was determined using the FACScanto II cytofluorimetry system (Becton Dickinson-BD, San Jose, CA, USA). In functional studies, CAFs and fibroblasts were used until the $5^{\text {th }}$ passage of culture.

\section{Cell phenotype analysis}

BM CAFs or fibroblasts from control patients were analyzed in whole heparinized aspirates. Cells were resuspended in lysing solution (BD) to lyse erythrocytes under gentle hypotonic conditions. Next, lysates were buffered in cytofix/cytoperm permeabilization buffer (BD) and incubated with a mouse anti-human aSMA-FITC monoclonal antibody (mAb) (Abcam, Cambridge, UK) and a rabbit anti-human FSP1 Ab (Sigma-Aldrich, St. Louis, MN, USA). Samples were then incubated with anti-rabbit PE- (R\&D Systems, Minneapolis, MN, USA) or APC-conjugated (Thermo Scientific, Rockford, IL, USA) antisera. Cell surface antigens were detected using the mAbs listed in Table S1, acquired by cytofluorimetry (FACScanto II, BD), and analyzed using FACSDiva software (BD). The negative controls included isotypematched, irrelevant antibodies.

\section{Cell cocultures and cell proliferation}

CAFs $\left(5 \times 10^{4}\right)$ were cocultured in RPMI-1640 medium (Euroclone, Pero, Milan, Italy) with autologous CD138 ${ }^{+}$plasma cells or RPMI8226 cells at a 1:1 or 10:1 cell ratio for 2 or 5 days. Parallel cocultures were performed using control fibroblasts/RPMI8226 cells. To evaluate the proliferation of each cell population, cocultured cells were labeled according to the manufacturers' instructions with two viable probes: carboxyfluorescein succinimidyl ester 
(CFSE, Invitrogen Corp., Carlsbād, CA,,USA) and Dye-eFluor-670 (e-Bioscience, San Diego,

CA, USA). For the transwell (0.4 $\mu \mathrm{m}$ pore size, Costar, Cambridge, MA, USA) experiments, CAFs were cultured in the lower chambers of 24-well plates, and MM cells were cultured in the upper chambers. CAFs and MM cells were also cultured individually to evaluate spontaneous proliferation. Samples were analyzed using the FACScanto II system. Results are presented as the percentage of proliferating cells and the "relative proliferation index" by comparing the cell proliferation in coculture to spontaneous proliferation.

\section{Apoptosis assay}

Apoptosis was assessed in CD138 ${ }^{+}$plasma cells using Annexin-V-FITC/7-aminoactinomycin$\mathrm{D}$ (7-AAD; BD-Pharmingen $)^{24}$.

\section{Chemotaxis, adhesion, and in vitro angiogenesis}

Chemotaxis was assessed using the Boyden microchamber assay. Adhesion of patient plasma cells or RPMI8266 cells to fibronectin (FN) and/or CAFs as well as the induction of in vitro angiogenesis (Matrigel $\left.{ }^{\circledR}, \mathrm{BD}\right)$ by CAFs were assessed ${ }^{24}$.

\section{In vivo mouse models}

The 5T33MM mouse. The mouse cell line 5T33MM was intravenously (i.v.) injected into 10 syngeneic C57B1/KaLwRij mice (Harlan CPB, Horst, The Netherlands) at 6-8 weeks of age. The development of MM was assessed by protein electrophoresis of the serum samples. When the serum paraprotein concentration achieved a level greater than $10 \mathrm{mg} \mathrm{mL}^{-1}$, mice were sacrificed, and BM from the femurs, tibiae, and humeri was flushed. Mononuclear cells were isolated by Lympholyte-M (Cedarlane, Hornby, Canada) gradient centrifugation, resuspended in RPMI-1640 medium supplemented with penicillin/streptomycin, glutamine, MEM, and sodium pyruvate (Gibco, Life Technologies, Gent, Belgium); and assayed for phenotype using the FACScanto II system. Mice were housed according to the Institutional Animal Care and Use Committee of the University of Brussels. 
MM xenograft mice. Twenty female, 6- ${ }^{-}$to 8 -week-old non-obese diabetic (NOD) severe combined immunodeficiency (scid) mice (NOD.CB17-Prkdcscid/NCrHsd, Harlan Laboratories, Udine, Italy) were injected subcutaneously into the left flank with RPMI8226 cells $\left(2\right.$ or $\left.5 \times 10^{6}\right)$ suspended in $100 \mu \mathrm{L}$ Hank's Balanced Salt Solution (HBSS, Euroclone) and $100 \mu \mathrm{L}$ Matrigel ${ }^{\circledR}$ (BD). Co-injection experiments were performed by admixing active MM CAFs with RPMI8226 cells at a 1:1 ratio. Tumor growth was measured twice weekly, and weights $\left(\mathrm{mg}=\mathrm{mm}^{3}\right)$ were calculated as the (length $[\mathrm{mm}] \times$ width $\left.^{2}\left[\mathrm{~mm}^{2}\right]\right) / 2$. Mice were sacrificed when the tumor weight was $\sim 2.5 \mathrm{~g}$. Mice were housed according to the Institutional Animal Care and Use Committee of the University of Bari Medical School.

\section{Matrigel plug assay}

A mixture containing growth factor-reduced Matrigel ${ }^{\circledR}(500 \mu \mathrm{L})$ and heparin $(40 \mathrm{U} / \mathrm{mL})$ was admixed with CAF conditioned medium (CM), $2 \times 10^{5}$ CAFs from active MM patients resuspended in HBSS medium, and either saline solution (negative control) or pro-angiogenic growth factors (200 ng/mL of each FGF2, VEGF, and HGF; positive control). Matrigel mixtures were injected subcutaneously into the abdominal midline of 6-week-old NOD/scid mice. Ten days after implantation, the animals were sacrificed, and the Matrigel plugs were harvested. Neovascularization was evaluated by immunofluorescence staining using an antiCD31-PE-conjugated mAb (BD-Pharmingen).

\section{Immunohistochemistry and immunofluorescence}

Immunohistochemistry and immunofluorescence analyses were performed on BM from MM and MGUS patients and 5T33MM mice as well as cultured cells. Details are supplied in the Supplementary Methods.

\section{Statistical analysis}

A statistical analysis was performed using GraphPad Prism 5 software. The data presented represent the mean \pm standard deviation $(\mathrm{SD})$. Results were analyzed using the Wilcoxon 
signed-rank test. $P<0.05$ was considered statistically significant. Survival curves were plotted using the Kaplan-Meier method.

\section{RESULTS}

\section{Phenotypic analysis of CAFs in MM and MGUS patients}

The gated CD45 population of BM CAFs was analyzed by cytofluorimetry (Figure 1a) for the expression of FSP1, $\alpha \mathrm{SMA}$, and FAP (Figure 1b-d). Based on FSP1 and aSMA expression, the following subpopulations were distinguished: $\mathrm{FSP}^{+} \alpha \mathrm{SMA}^{-}(\mathrm{P} 1), \mathrm{FSP}^{+} \alpha \mathrm{SMA}^{+}(\mathrm{P} 2)$, and $\mathrm{FSP}^{-} \alpha \mathrm{SMA}^{+}$(P3). Each of these subpopulations was significantly increased in patients with active MM (Figure 1b,c). As a whole, CAFs represented $75 \pm 20 \%$ of the CD45 cells in active MM patients compared with $18 \pm 7 \%$ and $33 \pm 12 \%$ in patients with nonactive MM and MGUS, respectively. In contrast, fibroblasts comprised only $9 \pm 5 \%$ of the CD45 cells in control patients. Representative dot plots are shown in Figure 1c. Analysis of FAP expression (selectively expressed on activated fibroblasts ${ }^{10,26}$ ) revealed that almost the entire gated $\mathrm{aSMA}^{+}$subpopulation (P2 plus P3) was $\mathrm{FAP}^{+}(70-99 \%)$ in active MM patients, whereas the gated $\mathrm{FSP}^{+}$subpopulation (P1) displayed variable proportions of $\mathrm{FAP}^{+}$cells $(20-98 \%)$ (Figure 1d). These data suggest that the $\mathrm{FSP}^{+}$cell population includes both resting and activated fibroblasts ${ }^{11}$. The increased frequency of $\alpha \mathrm{SMA}^{+} \mathrm{FSP} 1^{+} \mathrm{CAFs}$ in active MM patients was also demonstrated by the immunofluorescence analysis of cells selected with D7-FIBconjugated (anti-fibroblast) microbeads ${ }^{27}$ (Figure 1e). Overall, these results suggest that MM activity is associated with the expansion and activation of CAFs in the BM milieu. Indeed, the CM of active MM CAFs contained significantly increased levels of TGF $\beta$ (a major differentiation factor for fibroblasts ${ }^{16}$ ); SDF1 $\alpha$, IL-6, and IGF1 (key cytokines for MM progression $^{28}$ ); and VEGF and FGF2 (key angiogenic cytokines ${ }^{22,23}$ ) as compared to the CM of CAFs from nonactive MM, MGUS, and control patients (Figure 1f). Finally, the expression of specific markers of pericytes (NG2 and PDGFR $\beta$ ), endothelial cells (ECs; CD31, 
VEGFR2, and CD144), hematopoietic stem and progenitor cells (HSPCs; CD133), and mesenchymal stem cells (MSCs; CD146 and CD90) was analyzed for CAFs from MM and MGUS patients. CAFs from active MM patients showed phenotypic heterogeneity due to increased percentages of PDGFR $\beta^{+}(9-22 \%), \mathrm{CD}^{+} 1^{+}(5-25 \%), \mathrm{VEGFR}^{+}(3-15 \%), \mathrm{CD} 144^{+}$ (5-15\%), $\mathrm{CD} 133^{+}(1-3 \%)$, and $\mathrm{CD}^{+} 0^{+}(8-21 \%)$ cells as compared to CAFs from the other patient groups (Supplementary Figure 1).

\section{EndMT and MT contribute to the formation of CAFs}

Because active MM CAFs expressed EC markers (CD31 and VEGFR2), we investigated the possibility that these cells originate from ECs via TGF $\beta$-induced EndMT ${ }^{16}$. ECs from active MM patients were cultured for 5 days in CM from paired CAFs or RPMI8226 cells producing TGF $\beta$ (Figure 1f, Supplementary Figure 2). The addition of TGF $\beta$ served as a positive control, whereas cultures lacking TGF $\beta$ were used as a negative control. MM ECs treated with CAF CM, RPMI8226 cell CM, or TGF $\beta$ exhibited significantly increased expression of $\alpha$ SMA $(58 \pm 9 \%, 37 \pm 7 \%$ and $55 \pm 11 \%$, respectively, vs. $8 \pm 4 \%$ for the negative control) and FAP $(44 \pm 6 \%, 24 \pm 9 \%$ and $32 \pm 8 \%$, respectively, vs. $8 \pm 5 \%$ for the negative control). In contrast, increased expression of FSP1 (18 $\pm 7 \%)$ was only evident in active MM CAF CM (Figure 2a). The increased expression of $\alpha$ SMA, FAP, and FSP1 was also demonstrated by immunofluorescence (Figure 2b) and real-time RT-PCR (Figure 2c). Similar results, albeit to a lesser extent, were obtained by culturing ECs from MGUS patients with autologous CAF CM for 10 days (Supplementary Figure 3). Next, we examined whether CM from active MM CAFs and RPMI8226 cells could influence the in vitro angiogenesis of MM ECs (Figure 2d). Using a Matrigel ${ }^{\circledR}$ assay, MM ECs sprouted and aligned to form branching and anastomosed tubes with multicentric junctions, giving rise to a closely knit capillary plexus (control). However, treatment of MM ECs with TGF $\beta$, CAF CM, and, to a lesser extent, RPMI8226 cell $\mathrm{CM}$ reduced the number and thickness of these junctions (Figure 2d), signifying changes in vessel differentiation. Moreover, inhibition of the TGF $\beta$ pathway by the addition of the 
TGF $\beta$-receptor1 kinase $A$ inhibitor $\mathrm{SD} 208$ to $\mathrm{CAF} \mathrm{CM} /$ reduced aSMA expression

(Supplementary Figure 4a) and preserved the in vitro angiogenic activity (Supplementary Figure 4b) of ECs. Overall, our data suggest that CAFs from active MM patients may drive EndMT via TGF $\beta$.

Next, the effect of CAF and RPMI8226 cell CM on CD133 ${ }^{+}$HSPCs as precursors of ECs was analyzed. HSPCs were isolated from the peripheral blood of mobilized MM patients and grown on FN-coated plates in EC differentiation medium for 14 days ${ }^{29}$. Morphologically, the adherent HSPCs were heterogeneous due to the presence of small and large round cells as well as large flat cells (Figure 2e, control). As demonstrated by cytofluorimetry (Figure 2f), HSPCs were $\mathrm{CD} 34^{+} \mathrm{CD} 31^{+} \mathrm{CD} 144^{+}$VEGFR2 ${ }^{+}$, indicating commitment to the EC lineage, but were also $\mathrm{FSP}^{-} \alpha \mathrm{SMA}^{-} \mathrm{FAP}^{-}$(control). Exposure to active MM CAF CM and TGF $\beta$ for 7 days induced a drastic morphological transformation; cells became spindle-shaped (Figure 2e) and demonstrated increased expression of $\mathrm{FSP}^{+}(31 \pm 11 \%$ for $\mathrm{MM} \mathrm{CAF} \mathrm{CM}$ and $33 \pm 9 \%$ for TGF $\beta)$ and $\alpha \mathrm{SMA}^{+}(24 \pm 6 \%$ for MM CAF CM and $27 \pm 8 \%$ for TGF $\beta)$. These results indicated that CAF CM and TGF $\beta$ induced HSPC differentiation into CAF-like cells (Figure 2f), whereas CM from MGUS CAFs and control fibroblasts did not induce these changes (data not shown).

The origin of active MM CAFs from MSCs was also evaluated. BM MSCs from active MM patients were $\mathrm{CD} 146^{+} \mathrm{CD} 90^{+} \mathrm{CD} 105^{+} \mathrm{CD} 45^{-}$and $\mathrm{CD} 31^{-} \mathrm{CD} 34^{-}$(ECs and HSPCs markers) (Figure $2 \mathrm{~g}$ ), and these cells contained marginal proportions of $\mathrm{FSP}^{+}(6 \pm 3 \%), \alpha \mathrm{SMA}^{+}$ $(12 \pm 6 \%)$, and $\mathrm{FAP}^{+}(10 \pm 5 \%)$ cells (Figure $2 \mathrm{~g}$, control) that significantly increased after a 7 day culture with CM from autologous CAFs $(30 \pm 10 \%, 36 \pm 12 \%$, and $31 \pm 11 \%$, respectively), RPMI8226 cells $(23 \pm 8 \%, 30 \pm 12 \%$, and $29 \pm 9 \%$, respectively), and TGF $\beta(52 \pm 15 \%, 45 \pm 12 \%$, and $48 \pm 14 \%$, respectively) (Figure $2 \mathrm{~g}$ ). Immunofluorescence for FSP1 and $\alpha$ SMA confirmed the finding that active MM CAFs and RPMI8226 cells induced MSC transdifferentiation into CAF-like cells (Figure 2h). Because MSCs have a preferential tropism for tumor sites ${ }^{30}$, we 
investigated the migration of MSCs from active MM patients in response to paired $\mathrm{CAF}$ and RPMI8226 cell CM as well as IGF1 (positive control), and significant migration towards both types of CM was observed (Figure 2i).

Overall, these results suggest that active MM CAFs and MM cells induce ECs and HSPCs (via EndMT) as well as MSCs (via MT) to differentiate into CAFs.

\section{MM cells induce the proliferation of CAFs}

Dye-eFluor-labeled RPMI8226 cells were cocultured with CFSE-labeled CAFs from MM and MGUS patients or fibroblasts from control patients at a ratio of $1: 10$ or $1: 1$, either with or without a permeable transwell (Figure 3a,c,d, 'proliferation in coculture'). CAFs or fibroblasts were also cultured alone (Figure 3b, 'spontaneous proliferation'). CAF proliferation $(\mathrm{Q} 1+\mathrm{Q} 3)$ and FAP expression $(\mathrm{Q} 1+\mathrm{Q} 2)$ were evaluated after 5 days (Figure 3b,c). CAFs from active MM patients displayed significantly increased spontaneous proliferation (Figure 3b, Q1+Q3 $=25 \pm 6 \%$; Figure 3d) compared to those from patients with nonactive $\mathrm{MM}(10 \pm 3 \%)$ or MGUS $(7 \pm 4 \%)$ as well as fibroblasts from control patients $(5 \pm 2 \%)$. CAFs from active MM patients also exhibited significantly increased percentages of $\mathrm{FAP}^{+}$cells $(\mathrm{Q} 1+\mathrm{Q} 2=23 \pm 8 \%$ vs. $11 \pm 5 \%$ (nonactive $\mathrm{MM}), 7 \pm 3 \%$ (MGUS), and $<1 \%$ (control); Figure 3c,e). RPMI8226 cells enhanced both CAF proliferation and FAP expression (Figure 3c,d,e), and the relative proliferation index (Figure 3e) showed that the ability of RPMI8226 cells to induce CAF proliferation was independent of the disease state or FAP expression but was dependent on the number of MM cells (higher at 1:1 cell ratio) and cell-tocell contact (higher without transwell). However, physical separation of CAFs from MM cells by the addition of the transwell did not affect FAP expression.

\section{Active MM CAFs induce MM cell proliferation and prevent apoptosis}

Dye-eFluor-labeled CAFs from MM and MGUS patients or fibroblasts from control patients were cocultured with CFSE-labeled RPMI8226 cells or CD138 ${ }^{+}$plasma cells from active MM patients at 1:1 or 10:1 ratio, either with or without a permeable transwell, and proliferation 
was evaluated after 2 days. CAFs from active MM patients induced robust RPMI8226 cell proliferation, while $\mathrm{CD} 138^{+}$plasma cell proliferation was observed only at the 10:1 cell ratio, possibly because these cells were only minimally proliferative in vitro (Figure 4a). Moreover, this stimulatory effect was significantly reduced with the addition of the transwell (Figure 4a), implying a requirement for cell-to-cell contact.

The anti-apoptotic effect of CAFs was analyzed in the 1:1 and 10:1 cocultures with autologous $\mathrm{CD} 138^{+}$plasma cells, given that these stroma-dependent cells are more prone to spontaneous apoptosis than stroma-independent RPMI8226 cells. CD138 ${ }^{+}$cells were also cultured in serum-free medium (SFM) alone or in the presence of IGF1 plus IL-6 to prevent apoptosis $^{28}$. The percentage of cells undergoing spontaneous apoptosis was $48 \pm 8 \%$ in SFM, whereas it was only $8 \pm 3 \%$ in cultures with IGF1 plus IL-6 (Figure $4 \mathrm{~b}$ ). CAFs from active MM patients displayed an overlapping anti-apoptotic effect at both the 1:1 (12 $\pm 8 \%$ without transwell; $25 \pm 11 \%$ with) and $10: 1$ cell ratios (9 $5 \%$ without; $15 \pm 9 \%$ with). The results are expressed as the percentage of apoptosis observed in control cultures (Figure 4c) and demonstrated that CAFs from active MM significantly reduced spontaneous apoptosis. Because the anti-apoptotic effect was observed both with and without the transwell, cell contact-dependent and cytokine-dependent mechanisms may be involved. Overall, these data indicate that active MM CAFs prevent cell apoptosis, as demonstrated by a reduction in Annexin-V expression (Figure 4b), and drive MM cell proliferation, as measured by CFSE dilution (Figure 4a).

\section{SDF1 $\alpha$ and integrins mediate crosstalk between CAFs and MM cells}

The role of SDF1 $\alpha$ and its receptor CXCR4 in mediating crosstalk between CAFs and MM cells was investigated using AMD3100 (a specific CXCR4 inhibitor). The expression of SDF1 $\alpha$ and CXCR4 was previously shown to be elevated in CAFs in breast cancer ${ }^{7}$ and MM cells, respectively ${ }^{31}$. As shown in Figure 1f, significantly increased levels of SDF1 $\alpha$ were observed in the CAF CM from active MM patients compared to nonactive MM and MGUS 
patients. Moreover, CXCR4 $4^{+}$RPMI8226 cells and CD138 plasma cells from active MM patients migrated towards the CM from paired CAFs and SDF1 $\alpha$ cells (positive control, Figure 5a) and adhered to CAFs and FN (Figure 5b), whereas treatment of RPMI8226 cells and $\mathrm{CD}_{138^{+}}$plasma cells with AMD3100 reduced CXCR4 expression (Figure 5c). As specifically shown for RPMI8226 cells cocultured with CAFs, treatment with AMD3100 also inhibited migration (Figure 5d), adhesion (Figure 5e), and the relative proliferation index (Figure 5f).

Because integrins are known to govern interactions between BMSCs and MM cells ${ }^{32}$, the role of $\beta 1, \beta 3, \beta 7$, VLA4, VLA5, and $\alpha \mathrm{V} \beta 3$ integrins (major FN receptors) as well as cellular FN was analyzed (Figure 6). FN expression was significantly increased in CAFs from active MM patients $(70 \pm 12 \%)$ compared to nonactive MM and MGUS patients (44 $\pm 7 \%$ and $31 \pm 9 \%$, respectively; Figure 6a). FN was also highly expressed in $\mathrm{CD}_{138^{+}}$plasma cells from active MM patients (85 $12 \%)$ and RPMI8226 cells (55\%) (Figure 6a). Moreover, treatment of MM cells with anti-integrin and anti-FN blocking antibodies reduced their adhesion to CAFs. Only anti- $\beta 3,-\beta 7$, and $-\mathrm{FN}$ treatment of CAFs inhibited their adhesion to MM cells (Figure 6c), whereas treatment of $\mathrm{MM}$ cells with anti- $\beta 3,-\beta 7,-\mathrm{VLA} 4,-\alpha \mathrm{V} \beta 3$, and $-\mathrm{FN}$ produced inhibition of their adhesion to CAFs (Figure 6b). Overall, these data suggest that the interaction of MM cells with CAFs is mediated by several integrins as well as cell surface FN.

\section{CAFs and MM cells reciprocally sustain their proliferation in vivo}

We next investigated whether MM cells support CAF expansion as well as whether CAFs from active MM patients support MM tumor growth in syngeneic 5T33MM mice and tumor initiation in mice bearing $\mathrm{MM}$ xenografts. C57BlKaLwRijHsd mice received $0.5 \times 10^{6}$ 5T33MM cells i.v., and after 3 weeks, BM cells from 5T33MM-treated and naïve mice were analyzed for CAF content. The 5T33MM mice showed significantly increased proportions of $\mathrm{FSP}^{+} \alpha \mathrm{SMA}^{+} \mathrm{CD} 45^{-}$cells compared to naïve mice (Figure $7 \mathrm{a}, \mathrm{b}$ ), suggesting that MM cells induced substantial CAFs expansion in vivo. 
In addition, NOD/scid mice were injected subcutaneousty/with/RPMI8226 cells, CAFs

isolated from active MM patients, or a 1:1 mixture of $5 \times 10^{6} \mathrm{MM}$ cells and CAFs. The coinjection led to accelerated tumor growth, which began 35 days post-transplantation (Figure $7 \mathrm{c})$. The tumor weight at the $50^{\text {th }}$ day was significantly higher for mice receiving the coinjection as compared to the RPMI8226 plasmocytoma alone. The role of CAFs in MM tumor initiation was studied in mice injected with $2 \times 10^{6}$ RPMI8226 cells alone or in combination with CAFs at a 1:1 ratio, and the addition of CAFs significantly increased the development of plasmocytomas (Figure 7d). Specifically, 4/6 (67\%) coinjected mice developed plasmocytomas compared to only $1 / 6$ (17\%) mice injected with MM cells alone, and these data led us to hypothesize that CAFs sustain MM tumor growth and initiation.

Sections from RPMI8226 plasmocytoma xenografts both with and without CAFs were stained for CD31 to evaluate the angiogenic effect of CAFs (Figure 7e,f). Enhanced angiogenesis was observed in CAF-coinjected plasmocytomas (Figure 7e), which displayed a microvascular density (MVD) $\sim 3$ times greater than plasmocytomas injected alone (Figure 7f). This effect may be related to increased tumor burden, although experiments with Matrigel ${ }^{\circledR}$ plugs revealed that $\mathrm{CAFs}$ from active $\mathrm{MM}$ patients and their $\mathrm{CM}$ were able to attract mouse $\mathrm{CD} 31^{+}$ ECs into the plug and induce angiogenesis (Figure 7g). Additionally, the CAF CM behaved as a strong chemoattractant in vitro for MM ECs (Figure 7h). Overall, our data suggest that CAFs have a direct angiogenic effect, thereby potentially supporting MM tumor growth, at least partly, by promoting neovascularization.

\section{Immunohistochemistry of CAFs in the BM of MM and MGUS patients and mice}

BM sections from active MM patients displayed an increased frequency and more intense staining for FSP1 (Figure 8a) and $\alpha$ SMA (Figure 8b) cells as compared to those from MGUS patients. Similar images were obtained for the femur biopsies of 5T33MM mice vs. naïve mice (Figure 8d,e). MM cells (brown cells) were consistently found in close contact with $\mathrm{FSP}^{+}{ }^{+}$and $\alpha \mathrm{SMA}^{+} \mathrm{CAFs}$ (pink cells) in both MM patients and mice (Figure 8 , inserts). In 
MGUS biopsies, CAFs coexisted in close contact with plasma cells (Figure 8c, left), and no

FSP1- and $\alpha$ SMA-stained cells were observed in the absence of myeloma cells (Figure 8c, right). This finding is in agreement with our in vitro results showing the important role of cell-to-cell contact for MM cells and CAF expansion.

\section{DISCUSSION}

CAFs have been widely studied in solid tumors, where they are associated with high-grade histology and poor prognosis ${ }^{5-8,33,34}$, but not in hematological tumors such as MM. Here, CAFs were identified according to FSP1, $\alpha$ SMA, and FAP expression in BM samples from patients with MM or MGUS; fibroblasts from patients with deficiency anemia were studied as controls. The highest proportions of CAFs were found in active MM patients, suggesting that the MM activity phase involves the expansion of CAFs. No correlation between the percentage of CAFs and the D\&S stage was observed, which may be attributed to the following reasons. First, MM cell proliferation, and hence tumor burden, is induced not only by CAFs but also by other BMSCs, such as ECs and osteoclasts ${ }^{23}$. Second, CAFs demonstrate an elongated shape, which allows for contact with numerous MM cells (Figure 8), and a single CAF may therefore support the cell contact-induced proliferation of many MM cells. Similar results were obtained in 5T33MM mice, in which the increase in CAFs corresponded with MM development. Furthermore, immunohistochemistry analysis of BM from patients and mice confirmed that high levels of CAFs coexist with MM cells.

Similar to solid tumors ${ }^{10-12}$, the CAF population in MM was heterogeneous and expressed cell markers of ECs, HSPCs, and MSCs, suggesting their derivation from multiple cell lineages. Although the origin of CAFs is not well understood, one hypothesis suggests that they may derive from resident fibroblasts that differentiate into myofibroblasts by up-regulating $\alpha \mathrm{SMA}$ in response to signals from tumor cells ${ }^{35}$. In our study, MM cells were able to activate existing fibroblasts and recruit them via the secretion of TGF $\beta$. In this regard, MGUS or MM BM 
sections showed that FSP1 ${ }^{+}$and $\overline{\mathrm{SMA}}^{+} \mathrm{CAF}$ coexist only with MGUS or MM plasma cells.

Previous work has shown that human breast fibroblasts are converted into CAFs in vivo in step with tumor progression via the production of small amounts of TGF $\beta$ that prime the TGF $\beta$ autocrine signaling pathway ${ }^{21}$. Moreover, plasma cells and BMSCs from MM patients secrete high levels of TGF $\beta^{36}$, and TGF $\beta$ blockade suppresses MM cell growth and adhesion to $\mathrm{BMSCs}^{36,37}$, implying an important role for TGF $\beta$ in MM pathogenesis. CAFs, in turn, transform BM stroma by producing collagen and $\mathrm{FN}$ and secreting growth factors (TGF $\beta$, HGF, IGF1), cytokines (IL-1, IL-6), and chemokines (SDF1 $\alpha)^{4}$. In our study, CAFs from patients with active MM produced increased levels of TGF $\beta$, IGF1, IL- 6 , and SDF1 $\alpha$ compared to CAFs from nonactive MM, MGUS, and control patients. Moreover, in vitro experiments using a coculture system have shown that MM cells stimulate CAFs to produce cytokines through chemokine- and cell adhesion-mediated processes (manuscript in preparation). In addition, active MM CAFs have been shown to recruit ECs, HSPCs, and MSCs and induce them to undergo EndMT ${ }^{15-16}$ and $\mathrm{MT}^{14}$. Cell transdifferentiation may also increase the CAF component in the MM BM stroma. EndMT has been described in heart development $^{38}$, fibrosis, and cancer ${ }^{16,39}$, and $\mathrm{B} 16 \mathrm{~F} 10$ melanomas grown in transgenic mice were shown to induce $30 \%$ of ECs to transdifferentiate into CAFs coexpressing CD31 and FSP1 or $\alpha \mathrm{SMA}^{16}$. In our study, TGF $\beta$ and CM obtained from RPMI8226 cells and active CAFs from MM patients were able to convert ECs and HSPCs into CAF-like cells, in a manner similar to that observed in solid tumors ${ }^{40}$. Furthermore, BM MSCs may contribute to the generation of $\mathrm{CAFs}^{41}$. Indeed, the MSCs of active MM patients migrated toward the CM of RPMI8226 cells and active CAFs from MM patients and transdifferentiated into $\mathrm{FSP}^{+} \alpha \mathrm{SMA}^{+} \mathrm{FAP}^{+}$cells. Finally, MM cells induced the in vitro activation (via cytokines) and proliferation (via cell-to-cell contact) of CAFs. Overall, our data suggest that MM cells trigger changes in the BM microenvironment in terms of CAF number and activity. 


\section{CAFs from active MM patients support MM cell growth via the induction of proliferation by}

cell-to-cell contact and the prevention of apoptosis via both contact-dependent and independent (i.e., cytokine-mediated) pathways. These data are in agreement with other studies showing that the binding of MM cells to whole BMSCs triggers the secretion of cytokines promoting MM cell growth and survival ${ }^{42,43}$.

Studies in animal models have reported that CAFs promote cancer initiation and progression $^{7,8,21}$. Here, using an in vivo xenograft MM mouse model, animals coinjected with active MM CAFs and RPMI8226 cells showed accelerated tumor growth compared with those injected with RPMI8226 cells alone. Active MM CAFs also supported MM initiation, as mice coinjected with low number of RPMI8226 cells and active MM CAFs developed plasmocytomas, which was likely a consequence of both the anti-apoptotic and proliferative effects of CAFs; this response was not observed in mice injected with a low number of RPMI8226 cells alone. Immunohistochemistry of plasmocytoma xenografts and Matrigel plugs showed an angiogenic effect of active MM CAFs; indeed, CAFs may favor angiogenesis through the production of SDF1 $\alpha$, VEGF, and FGF2. Therefore, MM CAFs may contribute to the angiogenic switch and the subsequent angiogenic phase that parallels the transition of MGUS into $\mathrm{MM}^{44}$.

Recently, Ghobrial et al. ${ }^{28}$ emphasized the role of BM niches in MM initiation and diffusion by introducing the concept of 'premetastatic' and 'metastatic' niches. It is also plausible that MM cells use systems of cell dissemination similar to those regulating the cell trafficking of hematopoietic stem cells or the metastasis of carcinoma cells, a process that includes cell invasion, blood vessel entry, homing to distant foci as micrometastasis, and finally growth to macrometastasis. Metastasis is mediated by growth factors, cytokines, and/or exosomes of MM cells that up-regulate FN production by resident fibroblasts to promote the metastatic niche. Tentatively, we suggest an initial role for $\mathrm{MM}$ cells in the induction of $\mathrm{BM}$ micrometastatic niches through a contact-independent, cytokine-mediated conversion of 
resident fibroblasts into $\mathrm{CAFs}$, thereby allowing $\mathrm{CAFs}$ to prepare the metastatic vascular niche and undergo crosstalk with MM cells. We found that inhibition of the SDF1 $\alpha /$ CXCR4 axis affected MM cell migration, adhesion, and proliferation, indicating that MM CAFs recruit $\mathrm{CXCR} 4^{+} \mathrm{MM}$ cells via SDF1 $\alpha$ secretion to promote CAFs-to-MM cell adhesion and the reciprocal enhancement of cell proliferation and survival.

Integrins play a role in the pathophysiology of MM cells by mediating MM cell survival, proliferation, homing into and egress from the $\mathrm{BM}$, and cell adhesion-mediated drug resistance ${ }^{45-47}$. Although the intracellular mechanisms mediating these processes have not been fully elucidated, MM cell interactions with BMSCs and ECM induce cytoskeletal reorganization in $\mathrm{MM}$ cells to form 'adhesion plaques' via integrin clustering and the phosphorylation of FAK tyrosine kinase, which triggers the RAS/MAP kinase signal transduction cascade ${ }^{48}$. Here, using anti-integrin blocking $\mathrm{mAbs}$, we found that MM cell adhesion to CAFs is mediated through the reciprocal involvement of $\beta 3, \beta 7$, VLA4, VLA5, and $\alpha \mathrm{V} \beta 3$ integrins expressed on $\mathrm{MM}$ cells and $\beta 3$ and $\beta 7$ integrins expressed on CAFs. Whether these integrins contribute simultaneously or individually through synchronized temporal-spatial expression to trigger the reciprocal activities of MM cells and CAFs remains to be determined. Furthermore, the blockade of FN on MM cells and CAFs inhibited adhesion by $70 \%$ and $30 \%$, respectively, suggesting that cellular FN may act as an integrin receptor for the crosstalk between MM cells and CAFs. Previous studies have also shown that MM plasma cells produce $\mathrm{FN}^{49,50}$, which suggests that bidirectional interactions between $\mathrm{CAFs}$ and $\mathrm{MM}$ cells mediated by surface FN and $\beta 3, \beta 7$, VLA4, VLA5 and $\alpha \mathrm{V} \beta 3$ integrins may occur.

In conclusion, our results highlight an important interplay between CAFs and plasma cells during MM initiation and progression. Cancer cells induce and maintain the CAF activated phenotype, which, in turn, supports tumor progression by promoting ECM remodeling, cell proliferation, apoptosis resistance, and angiogenesis. Because these effects are mediated by cell adhesion molecules, growth factors, and chemokines released by both MM cells and 
CAFs, targeting CAFs in MM ${ }^{\text {can }}$ be envisaged as a novel and potentially effective therapeutic approach.

\section{ACKNOWLEDGMENTS}

This work was supported by the Associazione Italiana per la Ricerca sul Cancro (AIRC); an Investigator Grant (number 10099 to AV); the Special Program Molecular Clinical Oncology 5 per 1,000 (number 9965 to AV), Milan; the European Commission's Seventh Framework Programme (EU FPT7) OVER-MyR (number 278706 to AV and KV) and OPTATIO (number 278570 to DR); and the Ministry of Health (Progetto PRIN 2009 to RR and 2012 to AV), Rome, Italy. We are grateful to K. De Veirman (Vrije Universiteit Brussels) for help with the cytofluorimetric experiments in syngeneic 5T33 mice.

Conflict of interest disclosure: The authors declare no competing financial interests.

Supplementary information is available at the Leukemia website.

\section{REFERENCES}

1. Tlsty TD, Hein PW. Know thy neighbor: stromal cells can contribute oncogenic signals. Curr Opin Genet Dev 2001; 11: 54-59.

2. Li H, Fan X, Houghton J. Tumor microenvironment: the role of the tumor stroma in cancer. J Cell Biochem 2007; 101: 805-815.

3. Orimo A, Weinberg RA. Stromal fibroblasts in cancer: a novel tumor-promoting cell type. Cell Cycle 2006; 5: 1597-1601.

4. Kalluri R, Zeisberg M. Fibroblasts in cancer. Nat Rev Cancer 2006; 6: 392-401.

5. Franco OE, Shaw AK, Strand DW, Hayward SW. Cancer associated fibroblasts in cancer pathogenesis. Semin Cell Dev Biol 2010; 21: 33-39. 
6. Shimoda M, Mellody KT, Orimo A. Carcinoma-associated fibroblasts are a rate-limiting determinant for tumor progression. Semin Cell Dev Biol 2010; 21: 19-25.

7. Orimo A, Gupta PB, Sgroi DC, Arenzana-Seisdedos F, Delaunay T, Naeem R, et al. Stromal fibroblasts present in invasive human breast carcinomas promote tumor growth and angiogenesis through elevated SDF-1/CXCL12 Secretion. Cell 2005; 121: 335-348.

8. Erez N, Truitt M, Olson P, Arron ST, Hanahan D. Cancer-associated fibroblasts are activated in incipient neoplasia to orchestrate tumor-promoting inflammation in an NFkappaB-dependent manner. Cancer Cell 2010; 17: 135-147.

9. De Wever O, Demetter P, Mareel M, Bracke M. Stromal myofibroblasts are drivers of invasive cancer growth. Int J Cancer 2008; 123: 2229-2238.

10. Orimo A, Weinberg RA. Heterogeneity of stromal fibroblasts in tumors. Cancer Biol Ther 2007; 6: 618-619.

11. Sugimoto H, Mundel TM, Kieran MW, Kalluri R. Identification of fibroblast heterogeneity in the tumor microenvironment. Cancer Biol Ther 2006; 5: 1640-1646.

12. Anderberg C, Pietras K. On the origin of cancer-associated fibroblasts. Cell Cycle 2009; 8: $1461-1462$.

13. Hinz B, Phan SH, Thannickal VJ, Galli A, Bochaton-Piallat ML, Gabbiani G. The myofibroblasts: one function, multiple origins. Am J Pathol 2007; 170: 1807-1816.

14. Mishra PJ, Mishra PJ, Humeniuk R, Medina DJ, Alexe G, Mesirov JP, et al. Carcinomaassociated fibroblasts-like differentiation of human mesenchymal stem cells. Cancer Res 2008; 68: 4331-4339.

15. Direkze NC, Hodivala-Dilke K, Jeffery R, Hunt T, Poulsom R, Oukrif D, et al. Bone marrow contribution to tumor-associated myofibroblasts and fibroblasts. Cancer Res 2004; 64: 8492-8495. 
16. Zeisberg EM, Potentâ S, Xie L, Zeisberg M, Kalluri- R. Discovery of endothelial to mesenchymal transition as a source for carcinoma-associated fibroblasts. Cancer Res 2007; 67: 10123-10128.

17. Kalluri R, Weinberg RA. The basics of epithelial-mesenchymal transition. J Clin Invest 2009; 119: 1420-1428.

18. Hinz B, Gabbiani G. Mechanisms of force generation and transmission by myofibroblasts. Curr Opin Biotechnol 2003; 14: 538-546.

19. Tschumperlin DJ, Dai G, Maly IV, Kikuchi T, Laiho LH, McVittie AK, et al. Mechanotransduction through growth-factor shedding into the extracellular space. Nature 2004; 429: 83-86.

20. Kumar S, Weaver VM. Mechanics, malignancy, and metastasis: the force journey of a tumor cell. Cancer Metastasis Rev 2009; 28: 113-127.

21. Kojima Y, Acar A, Eaton EN, Mellody KT, Scheel C, Ben-Porath I, et al. Autocrine TGFbeta and stromal cell-derived factor-1 (SDF-1) signaling drives the evolution of tumorpromoting mammary stromal myofibroblasts. Proc Natl Acad Sci USA 2010; 107: 2000920014.

22. Ria R, Reale A, De Luisi A, Ferrucci A, Moschetta M, Vacca A. Bone marrow angiogenesis and progression in multiple myeloma. Am J Blood Res 2011; 1: 76-89.

23. Vacca A, Ribatti D. Bone marrow angiogenesis in multiple myeloma. Leukemia 2006; 20: 193-199.

24. Basile A, Moschetta M, Ditonno P, Ria R, Marech I, De Luisi A, et al. Pentraxin 3 (PTX3) inhibits plasma cell/stromal cell cross-talk in the bone marrow of multiple myeloma patients. J Pathol 2013; 229: 87-98.

25. Berardi S, Caivano A, Ria R, Nico B, Savino R, Terracciano R, et al. Four proteins governing overangiogenic endothelial cell phenotype in patients with multiple myeloma are plausible therapeutic targets. Oncogene 2012; 31: 2258-2269. 
26. Santos AM, Jung J, Aziz N, Kissil JL, Purè E. Targeting fibroblast activation protein inhibits tumor stromagenesis and growth in mice. J Clin Invest 2009; 119: 3613-3625.

27. Jones EA, Kinsey SE, English A, Jones RA, Straszynski L, Meredith DM, et al. Isolation and characterization of bone marrow multipotential mesenchymal progenitor cells. Arthritis Rheum 2002; 46: 3349-3360.

28. Ghobrial IM. Myeloma as a model for the process of metastasis: implications for therapy. Blood 2012; 120: 20-30.

29. Ria R, Piccoli C, Cirulli T, Falzetti F, Mangialardi G, Guidolin D, et al. Endothelial differentiation of hematopoietic stem and progenitor cells from patients with multiple myeloma. Clin Cancer Res 2008; 14: 1678-85.

30. Xu S, Menu E, De Becker A, Van Camp B, Vanderkerken K, Van Riet I. Bone marrowderived mesenchymal stromal cells are attracted by multiple myeloma cell-produced chemokine CCL25 and favor myeloma cell growth in vitro and in vivo. Stem Cells 2012; 30: $266-279$.

31. Möller C, Strömberg T, Juremalm M, Nilsson K, Nilsson G. Expression and function of chemokine receptors in human multiple myeloma. Leukemia 2003; 17:203-210.

32. Damiano JS, Dalton WS. Integrin-mediated drug resistance in multiple myeloma. Leuk Lymphoma 2000; 38: 71-81.

33. Saito RA, Micke P, Paulsson J, Augsten M, Peña C, Jönsson P, et al. Forkhead box F1 Regulates tumor-promoting properties of cancer-associated fibroblasts in lung cancer. Cancer Res 2010; 70: 2644-2654.

34. Ko SY, Barengo N, Ladanyi A, Lee JS, Marini F, Lengyel E, et al. HOXA9 promotes ovarian cancer growth by stimulating cancer-associated fibroblasts. J Clin Invest 2012; 122: $3603-3617$.

35. Östman A, Augsten M. Cancer-associated fibroblasts and tumor growth-bystanders turning into key players. Curr Opin Genet Dev 2009; 19: 67-73. 
36. Urashima M, Ogata A,C Chauhan=D, Hatziyanni M, Vidriales MB, Dedera DA, et al. Transforming growth factor-beta1: differential effects on multiple myeloma versus normal B cells. Blood 1996; 87: 1928-38.

37. Hayashi T, Hideshima T, Nguyen AN, Munoz O, Podar K, Hamasaki M, et al. Transforming growth factor beta receptor I kinase inhibitor down-regulates cytokine secretion and multiple myeloma cell growth in the bone marrow microenvironment. Clin Cancer Res 2004; 10: 7540-7546.

38. Markwald RR, Fitzharris TP, Smith WN. Sturctural analysis of endocardial cytodifferentiation. Dev Biol 1975; 42: 160-180.

39. van Meeteren LA, ten Dijke P. Regulation of endothelial cell plasticity by TGF- $\beta$. Cell Tissue Res 2012; 347: 177-186.

40. Moonen JR, Krenning G, Brinker MG, Koerts JA, van Luyn MJ, Harmsen MC. Endothelial progenitor cells give rise to pro-angiogenic smooth muscle-like progeny. Cardiovasc Res 2010; 86: 506-515.

41. Spaeth EL, Dembinski JL, Sasser AK, Watson K, Klopp A, Hall B, et al. Mesenchymal stem cell transition to tumor-associated fibroblasts contributes to fibrovascular network expansion and tumor progression. PLoS One 2009; 4: e4992.

42. Wang LH, Yang XY, Zhang X, Farrar WL. Inhibition of adhesive interaction between multiple myeloma and bone marrow stromal cells by PPARgamma cross talk with NFkappaB and C/EBP. Blood 2007; 110: 4373-4384.

43. Teoh G, Anderson KC. Interaction of tumor and host cells with adhesion and extracellular matrix molecules in the development of multiple myeloma. Hematol Oncol Clin North Am 1997; 11: 27-42.

44. Ribatti D, Nico B, Crivellato E, Roccaro AM, Vacca A. The history of the angiogenic switch concept. Leukemia 2007; 21: 44-52. 
45. Damiano JS, Hazlehurst LA, Dalton WS. Cell adhesion-mediated drug resistance (CAMDR) protects the K\%\&" chronic mielogenous leukemia cell line from apoptosis induced by BCR/ABL inhibition, cytotoxic drugs, and gamma-irradiation. Leukemia 2001; 15:1232-1239.

46. Damiano JS. Integrins as novel drug targets for overcoming innate drug resistance. Curr Cancer Drug Targets 2002; 2: 37-43.

47. Nefedova Y, Landowski TH, Dalton WS. Bone marrow stromal-derived soluble factors and direct cell contact contribute to de novo drug resistance of myeloma cells by distinct mechanisms. Leukemia 2003; 17:1175-1182.

48. Vacca A, Ria R, Presta M, Ribatti D, Iurlaro M, Merchionne F, et al. alpha(v)beta(3) integrin engagement modulates cell adhesion, proliferation, and protease secretion in human lymphoid tumor cells. Exp Hematol 2001; 29: 993-1003.

49. van Riet I, de Greef C, del Favero H, Demanet C, Van Camp B. Production of fibronectin and adherence to fibronectin by human myeloma cell lines. Br J Haematol 1994; 87: 258265.

50. Tancred TM, Belch AR, Reiman T, Pilarski LM, Kirshner J. Altered expression of fibronectin and collagens I and IV in multiple myeloma and monoclonal gammopathy of undetermined significance. J Histochem Cytochem 2009; 57: 239-247. 


\section{ACCEPTEDLEGENDSE PREVIEW}

\section{Figure 1. Analysis of BM CAFs}

(a) Cytofluorimetric analysis of BM CAFs on gated CD45- cells and (b) the distribution of the $\mathrm{FSP}^{+} \alpha \mathrm{SMA}^{-}$(blue, P1), $\mathrm{FSP}^{+} \alpha \mathrm{SMA}^{+}$(red, $\mathrm{P} 2$ ), and $\mathrm{FSP}^{-} \alpha \mathrm{SMA}^{+}$(green, P3) subpopulations in 23 patients with active MM, 15 with nonactive MM, 18 with MGUS, and 11 patients with deficiency anemia (controls). $* P<0.05 ; * * P<0.01 v s$. the other groups. (c) $\mathrm{FSP}^{+}$and $\alpha \mathrm{SMA}^{+}$expression in representative patients. (d) Analysis of FAP expression on gated $\mathrm{FSP}^{+} \alpha \mathrm{SMA}^{-}$(blue, P1), $\mathrm{FSP}^{+} \alpha \mathrm{SMA}^{+}$(red, P2), and $\mathrm{FSP}^{-} \alpha \mathrm{SMA}^{+}$(green, P3) subpopulations in representative patients. Histogram markers were created based on the isotype negative control (data not shown). (e) Immunofluorescence staining of purified BM CAFs with $\alpha$ SMA (green) and FSP1 (red) in representative MM, MGUS, and control patients. Cell nuclei were stained with DAPI. Original magnification $200 \mathrm{X}$, bar $=50 \mu \mathrm{m}$. (f) TGF $\beta$, SDF1 $\alpha$, IL-6, IGF1, VEGF, and FGF2 levels were assessed by ELISA in the CM from CAFs obtained from 17 active MM, 15 nonactive MM, 13 MGUS, and 10 control patients. Values are expressed as the mean $\pm \mathrm{SD}$. $* * P<0.01 v s$. the other groups.

\section{Figure 2. EndMT and MT contribute to CAF generation}

(a-d) ECs from 7 active MM patients were grown in DMEM medium (negative control), CM from paired CAFs or RPMI8226 cells, or TGF $\beta$ (10 ng/mL, positive control) for 5 days. (a) Cytofluorimetry of VEGFR2 and CD31 expression (EC markers) as well as FSP1, $\alpha$ SMA, and FAP expression (CAF markers). (b) Representative immunofluorescence of FSP1 (red) and $\alpha$ SMA (green); cell nuclei were stained with DAPI. Original magnification 400X, bar = $25 \mu \mathrm{m}$. (c) Real-time RT-PCR results for FSP1, aSMA, and FAP mRNA expression (normalized to $G A P D H$ ) expressed as the mean \pm SD. $* P<0.05$, $* * P<0.01 v s$. negative control. Note the increase in $\alpha \mathrm{SMA}$ and FAP mRNA and protein expression upon the exposure of MM ECs to the CM of CAFs and RPMI8226 cells as well as TGF $\beta$. (d) In vitro angiogenic 
activity of MM ECs from a representative patient at initial diagnosis. Note that CAF and RPMI8226 cell CM as well as TGF $\beta$ reduced the number and thickness of vessel junctions. Original magnification 200X, bar $=50 \mu \mathrm{m}$. (e-f) $\mathrm{CD} 133^{+} \mathrm{HSPCs}$ from $7 \mathrm{MM}$ patients grown on FN-coated plates in EC differentiation medium for 14 days, followed by culture in the same medium alone (negative control), with TGF $\beta$ (10 ng/mL, positive control), or the CM from paired CAFs or RPMI8226 cells. (e) Adherent HSPCs were either small and round or flat and elongated in the control and RPMI8226 CM. In the CAF CM and TGF $\beta$ samples, the cells were spindle-shaped and similar to fibroblast-like cells (arrows). Original magnification 200X, bar $=50 \mu \mathrm{m}$. (f) Adherent HSPCs demonstrated increased FSP1, $\alpha$ SMA, and FAP expression upon exposure to $\mathrm{CAF} \mathrm{CM}$ and TGF $\beta$. (g-i) $\mathrm{CD} 146^{+} \mathrm{CD} 105^{+} \mathrm{CD} 90^{+} \mathrm{CD} 34^{-} \mathrm{CD} 31^{-}$ CD45 ${ }^{-}$MSCs from 9 active MM patients were cultured for 5 days with DMEM medium (negative control), the CM from paired CAFs or RPMI8226 cells, or TGF $\beta(10 \mathrm{ng} / \mathrm{mL}$, positive control). (g) Cytofluorimetric analysis revealed increased FSP1, $\alpha$ SMA, and FAP expression in MSCs treated with CAF and RPMI8226 cell CM and TGF $\beta$. (h) Representative immunofluorescence for $\alpha$ SMA (green) and FSP1 (red); cell nuclei were stained with DAPI. Original magnification $400 \mathrm{X}$, bar $=25 \mu \mathrm{m}$. (i) In vitro migration of $\mathrm{CD} 146^{+}$cells toward paired CAF and RPMI8226 cell CM (IGF1 = positive control). Values are expressed as the mean $\pm \mathrm{SD} ; * P<0.05 ; * * P<0.01$ vs. serum-free DMEM (SFM).

\section{Figure 3. RPMI8226 cells induce CAF proliferation and activation}

CAFs purified from 18 active MM, 12 nonactive MM, 12 MGUS, and 8 control patients were labeled with CFSE and cocultured with Dye-eFluor-stained RPMI8226 cells for 5 days. (a-c) Cytofluorimetry of FAP expression (Q1+Q2) and cell proliferation $(\mathrm{Q} 1+\mathrm{Q} 3)$ among $(\mathbf{a})$ gated CFSE-labeled CAFs. Representative proliferation (CFSE) and FAP profiles of (b) CAFs cultured in medium alone ("spontaneous" proliferation and FAP expression) and (c) CAFs cocultured at a ratio of $1: 1$ or $10: 1$ with RPMI8226 cells with or without a transwell insert. 
Decreased CFSE fluorescence intensity indicates proliferating cells. (d) Proliferating CAFs from all patients are expressed as the mean $\pm \mathrm{SD}$; ** $P<0.01 v s$. the other groups. (e) CAF proliferation was measured according to the relative proliferation index and FAP expression in all patients and is expressed as the mean $\pm \mathrm{SD} ; * * P<0.01 v s$. CAFs alone.

\section{Figure 4. Effect of CAFs on MM cell proliferation and apoptosis}

(a) CFSE-stained RPMI8226 cells and CD138 ${ }^{+}$plasma cells from MM and MGUS patients were cocultured at a 1:1 or 1:10 ratio for 2 days with Dye-eFluor-stained CAFs from 12 active MM, 10 nonactive MM, 7 MGUS, and 5 control patients with or without a transwell insert. The CAF-induced proliferation of RPMI8226 and CD138 ${ }^{+}$cells was expressed as the relative proliferation index, with values representing the mean \pm SD. (b, c) $\mathrm{CD} 138^{+}$plasma cells from 12 active MM patients were cultured in SFM ('spontaneous' apoptosis) with IL-6 plus IGF1 (both at $100 \mathrm{ng} / \mathrm{mL}$ ) or with CAFs as described above. Apoptosis was evaluated according to Annexin-V/7-AAD staining. (b) CAFs prevented the spontaneous apoptosis of CD138 ${ }^{+}$ plasma cells in a manner similar to treatment with IL-6 plus IGF1 in a representative MM patient. (c) Apoptotic CD138 ${ }^{+}$plasma cells were evaluated as the percentage of spontaneous apoptotic cells in all patients and are presented as the mean \pm SD. $* P<0.05$, ** $P<0.01$ vs. the other groups.

Figure 5. Involvement of the SDF1 1 /CXCR4 axis in the interaction between CAFs and MM cells

(a) Chemotaxis of $\mathrm{CD}_{138^{+}}$cells purified from 12 active MM patients and RPMI8226 cells towards SFM, CAF CM obtained from 12 active MM, 10 nonactive MM, 7 MGUS patients, and SFM+SDF1 $\alpha(100 \mathrm{ng} / \mathrm{mL})$. Values are expressed as the mean $\pm \mathrm{SD} ; * P<0.05, * * P<0.01$ vs. SFM. (b) Adhesion of CD138 ${ }^{+}$cells purified from 12 active MM patients and RPMI8226 cells to bovine serum albumin (BSA, negative control), FN, and CAFs from 12 active MM, 
10 nonactive MM, and 7 MGUS patients. Values are expressed as the mean $\pm \mathrm{SD} ; * P<0.05$, $* * P<0.01$ vs. BSA. Effect of AMD3100 treatment on (c) CXCR4 expression in RPMI8226 and CD138 ${ }^{+}$cells; (d) RPMI8226 cell migration; (e) RPMI8226 cell adhesion expressed as a percentage of untreated (control) cells; and (f) proliferation expressed as the relative proliferation index; $* * P<0.01 v s$. untreated cells.

\section{Figure 6. Involvement of integrins and FN in the crosstalk between CAFs and MM cells}

(a) Surface expression of $\mathrm{FN}$ on $\mathrm{CD} 138^{+}$plasma cells from 12 active $\mathrm{MM}$ patients, RPMI8226 cells, and CAFs from 12 active MM patients, 10 nonactive MM patients, and 7 MGUS patients. A representative cytofluorimetric histogram for each group of patients is shown. (b) Adhesion of RPMI8226 cells upon treatment with blocking mAbs to each indicated integrin on active MM CAFs from 8 patients. (c) Adhesion of untreated RPMI8226 cells on active MM CAFs from the 8 patients upon treatment with the indicated mAbs. Values are expressed as the mean $\pm \mathrm{SD} ; * P<0.05$, $* * P<0.01$ vs. control.

\section{Figure 7. In vivo interactions between CAFs and MM cells}

(a-d) The reciprocal effects of CAFs and MM cells in syngeneic and xenograft mice. (a) Cytofluorimetry of FSP 1 and $\alpha$ SMA expression in $95 \mathrm{~T} 33 \mathrm{MM}$ and 8 naïve mice; $* * P<0.01$. (b) Dot plots from representative mice. Note the CAF expansion in 5T33MM mice. (c-d) NOD/scid mice bearing xenografts generated from RPMI8226 cells, CAFs from active MM patients, or a 1:1 mixture of RPMI8226 cells and CAFs. The plasmocytoma growth curves were evaluated by tumor weight. Note the favorable effect of CAFs on both tumor growth (c) and establishment (d); $* P<0.05$, $* * P<0.01$ vs. RPMI8226 cells alone. (e-g) The angiogenic effect of CAFs. (e) CD31-stained microvessels in plasmocytomas. Note the enhanced angiogenesis in plasmocytomas with CAFs; neovessels are marked by arrowheads. (f) Increased microvessel counts in plasmocytomas with CAFs; $* * P<0.01$ vs. plasmocytomas 
alone. (g) The Matrigel plug assay revealed the ability of active MM CAFs and CM to attract mouse $\mathrm{CD}_{3} 1^{+}$neovessels. Original magnification $200 \mathrm{X}$, bar $=50 \mu \mathrm{m}$. (h) In vitro migration of MM ECs towards CAF CM vs. the negative control. Values are expressed as the mean \pm SD of 5 independent experiments; $* * P<0.01 v s$. SFM.

\section{Figure 8. Immunohistochemistry of CAFs in the BM of mice and patients}

(a, b) Plasma cells (brown) as well as $\mathrm{FSP}^{+}$and $\alpha \mathrm{SMA}^{+} \mathrm{CAFs}$ (pink) were increased and in close contact in an active MM patient (inserts). (c) In a MGUS patient, CAFs coexisted with plasma cells (left) but were not found without plasma cells (right). (e, f) 5T33MM mice exhibited similar findings compared to healthy mice. Original magnifications: $400 \mathrm{X}$, scale bar $=25 \mu \mathrm{m}$; inserts $600 \mathrm{X}$, scale bar $=16.6 \mu \mathrm{m}$. 

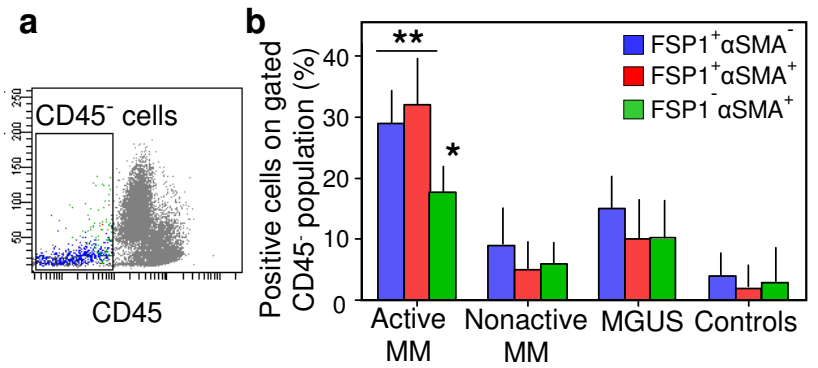

c

C $1^{\text {st }}$ Diagnosis MM $1^{\text {st }}$ Diagnosis MM Relapsed MM
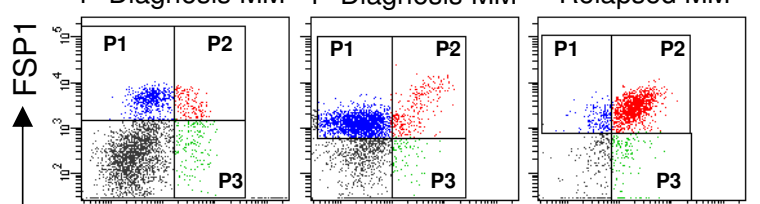

Remission MM

MGUS

Control

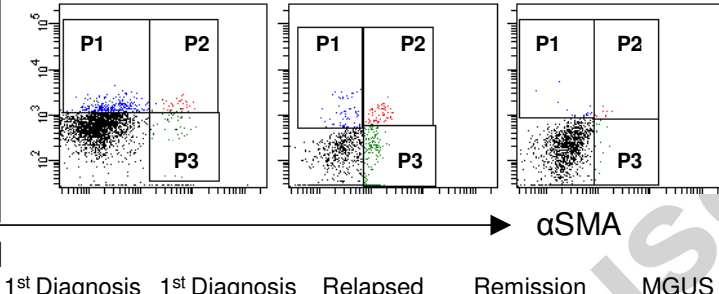

$1^{\text {st }}$ Diagnosis $1^{\text {st }}$ Diagnosis Relapsed Remission MGUS
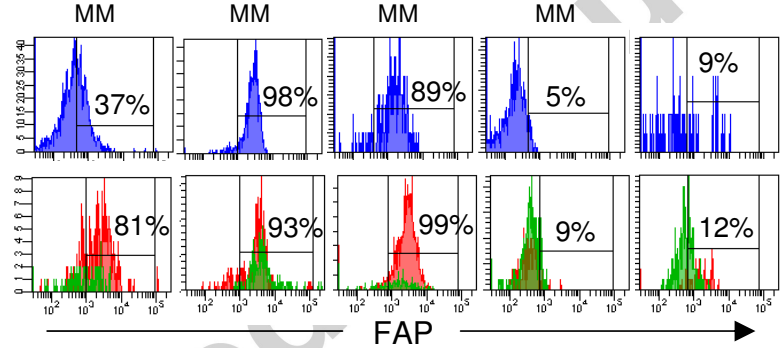

e

1st Diagnosis Remission MM

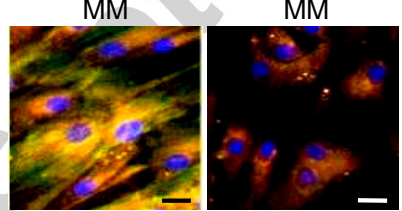

MGUS

Control

FSP1/aSMA/DAPI
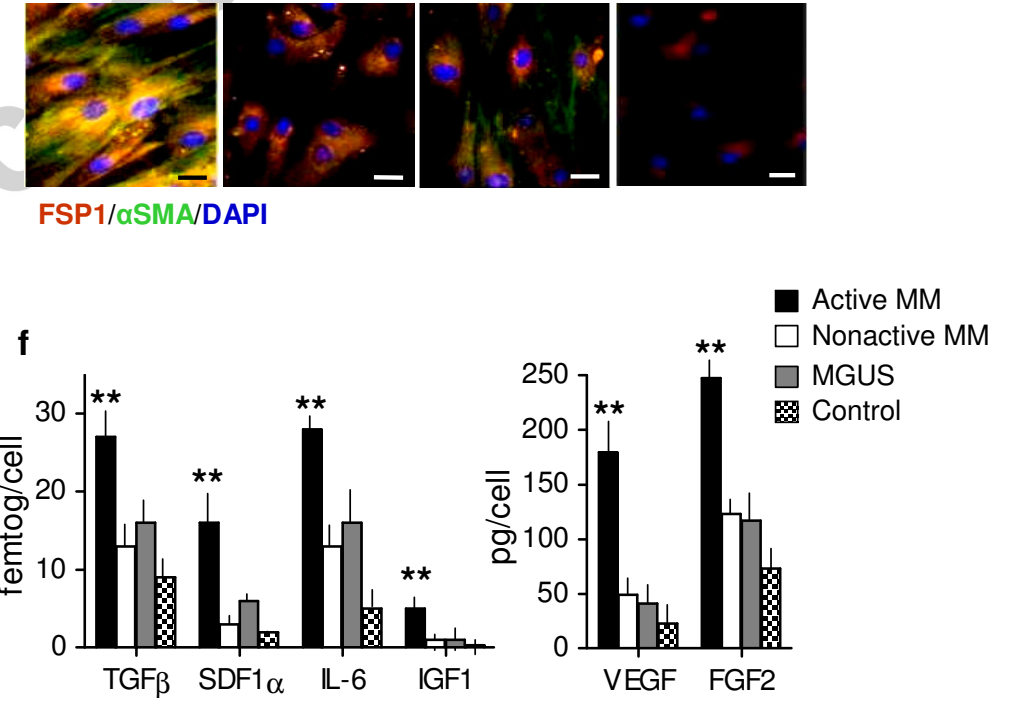

\section{Frassanito et al. Figure 1}



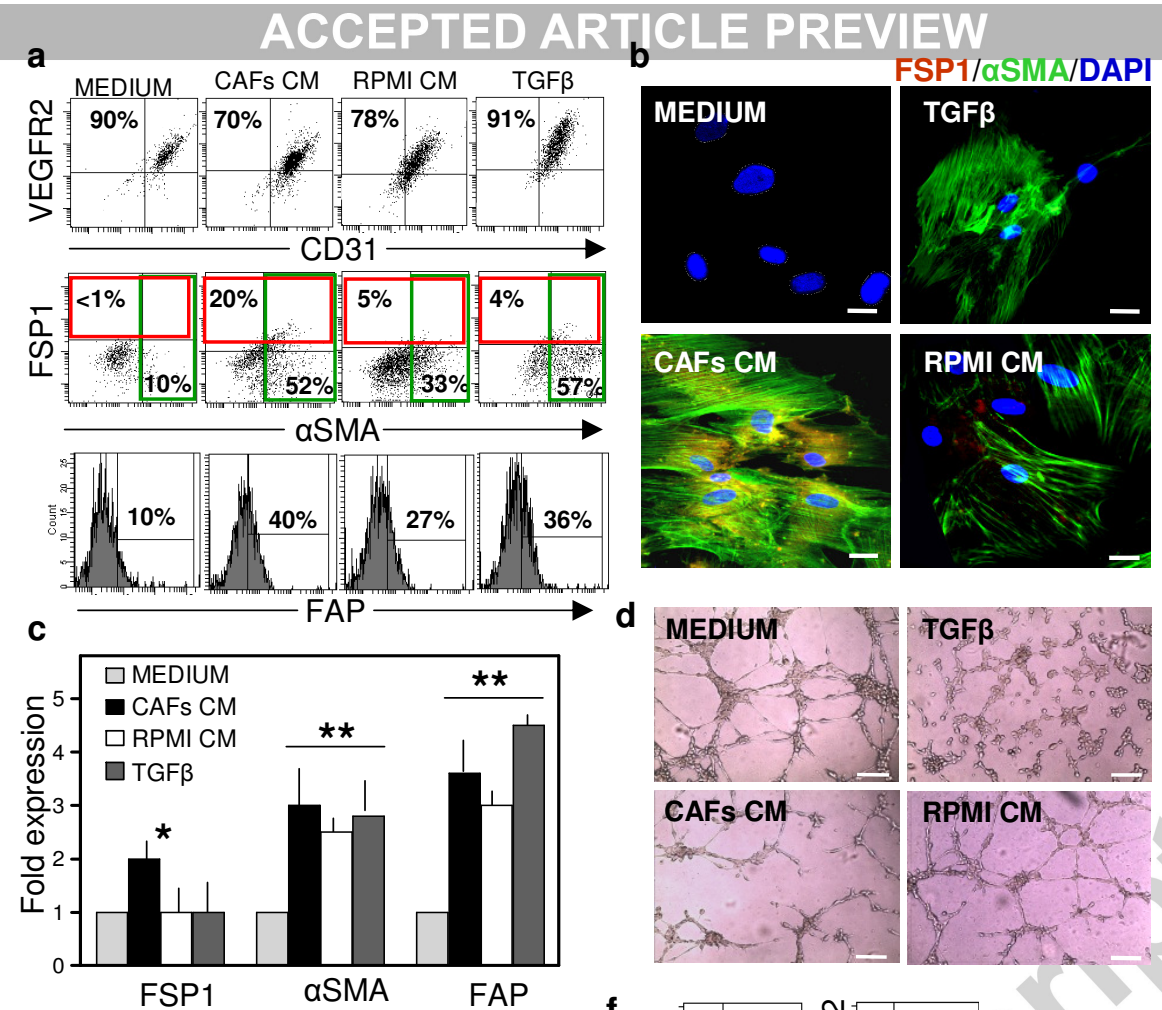

e
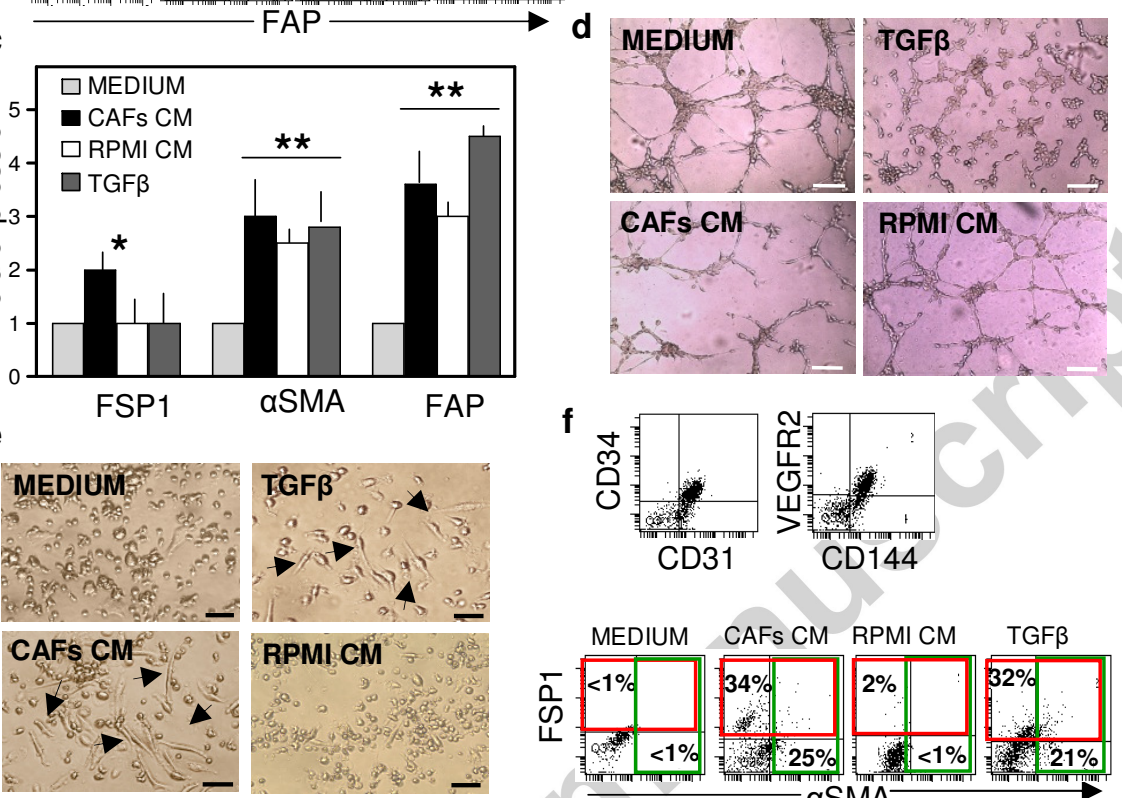

g
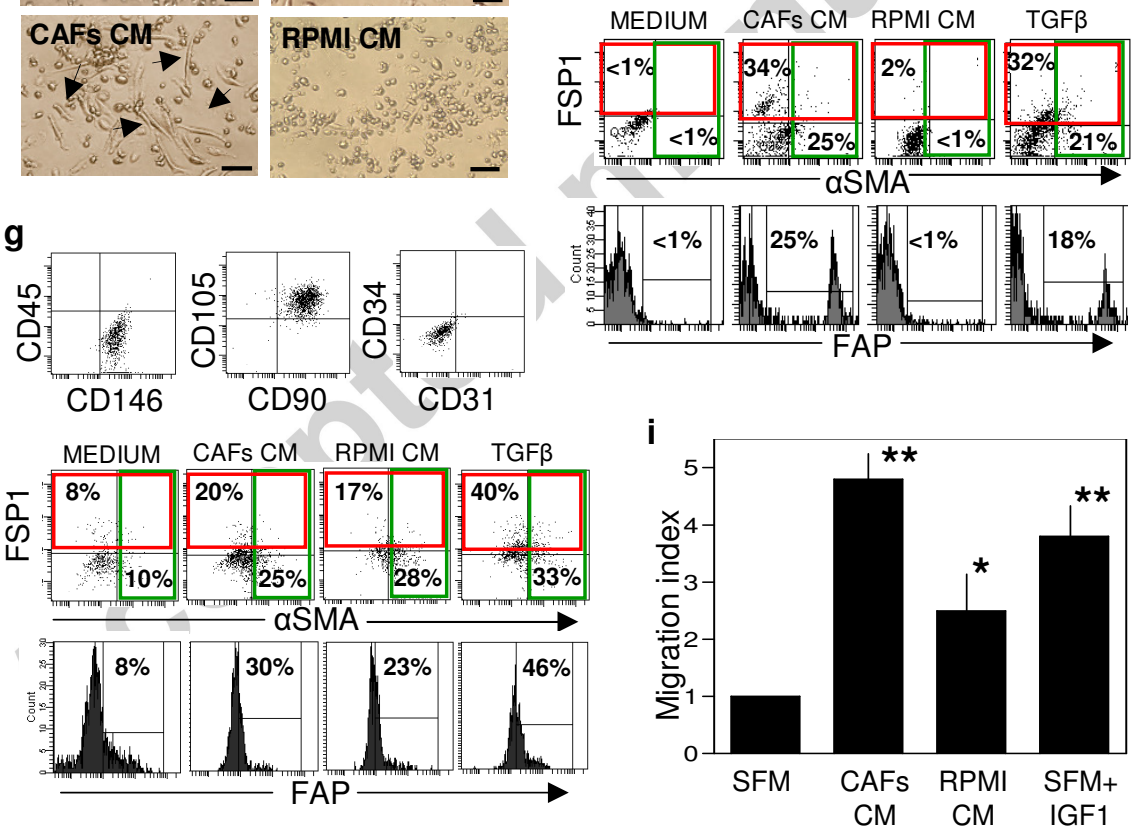

FSP1/aSMA/DAPI
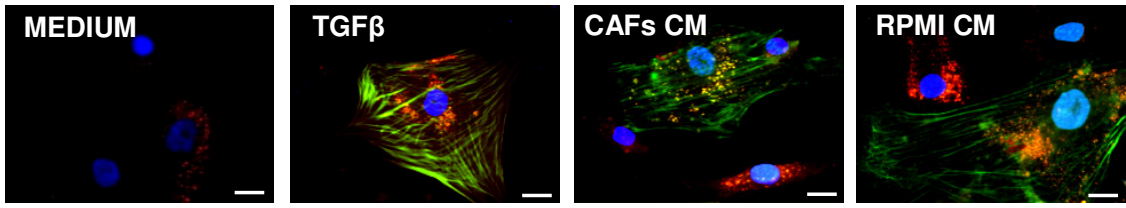

Frassanito et al. Figure 2 

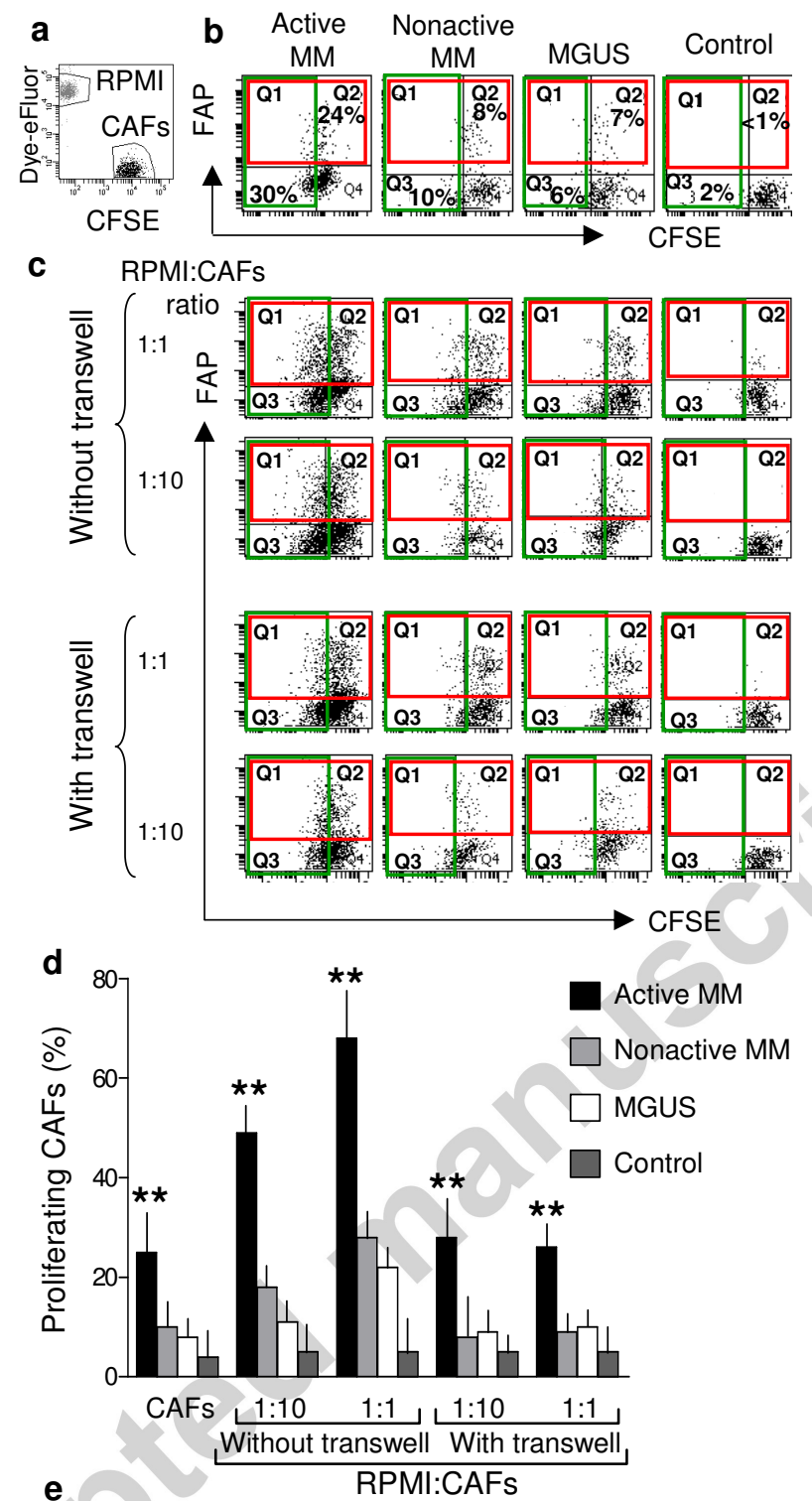

e
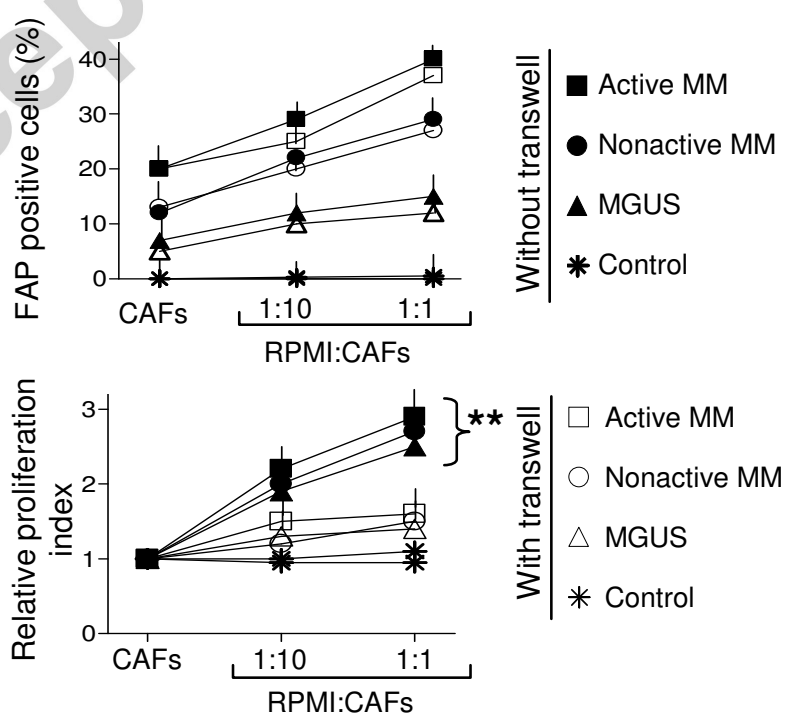

Frassanito et al. Figure 3 

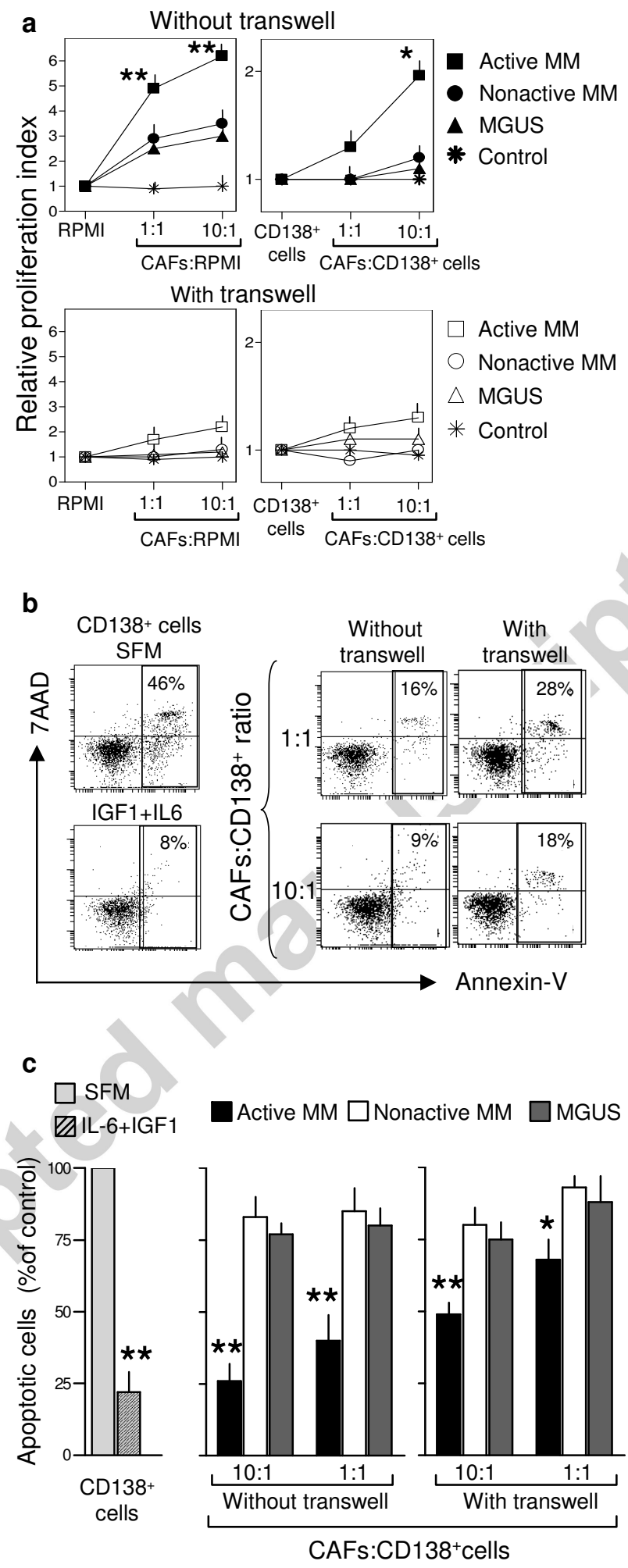

\section{Frassanito et al. Figure 4}




\section{ACCEPTED ARTICLE PREVIEW}
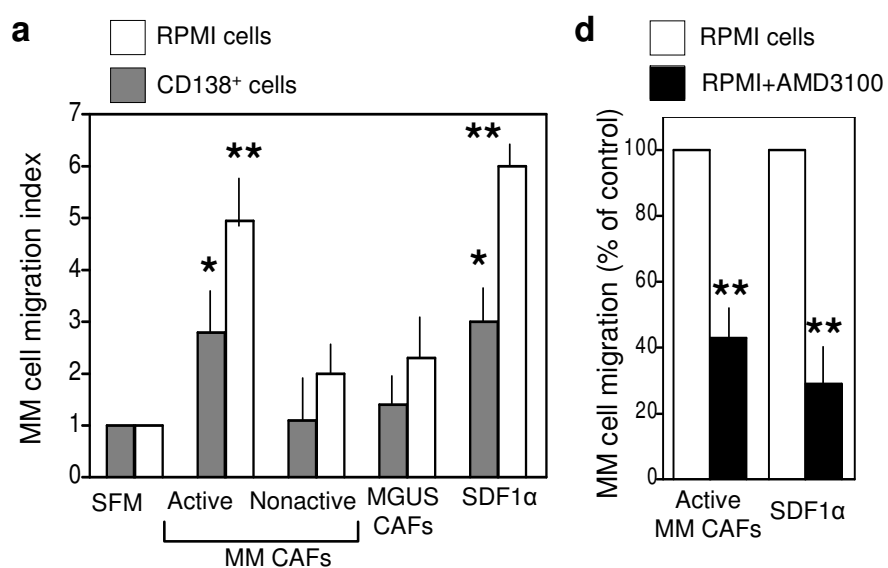

b
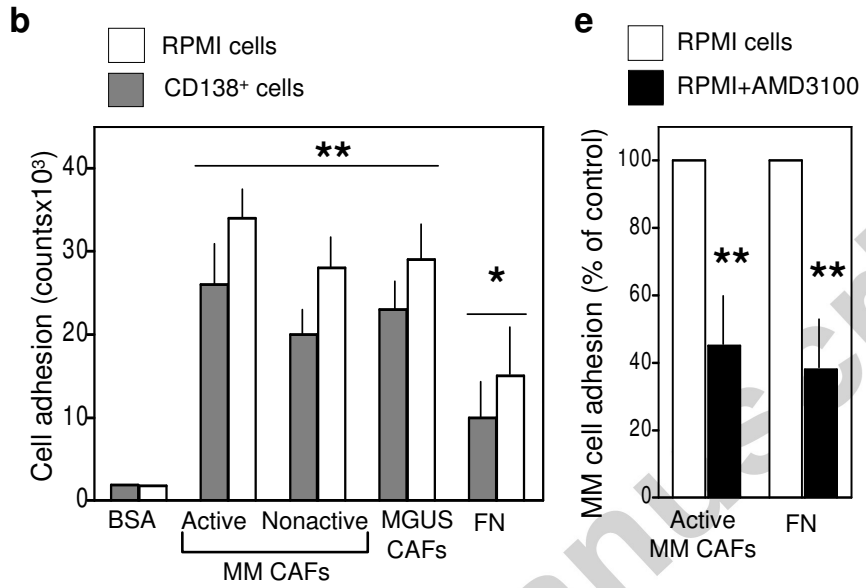

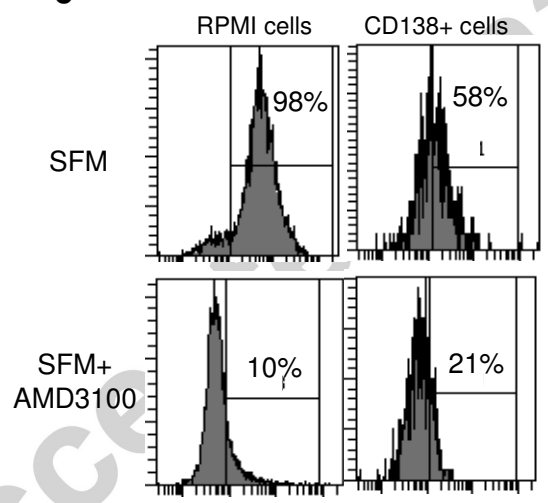

CXCR4 f

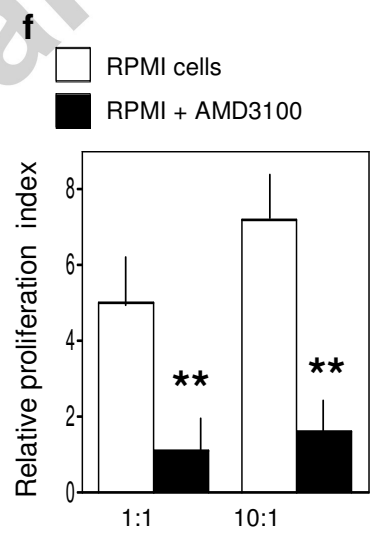

CAFs:RPMI cells

\section{Frassanito et al. Figure 5}


a

RPMI cells CD138+cells
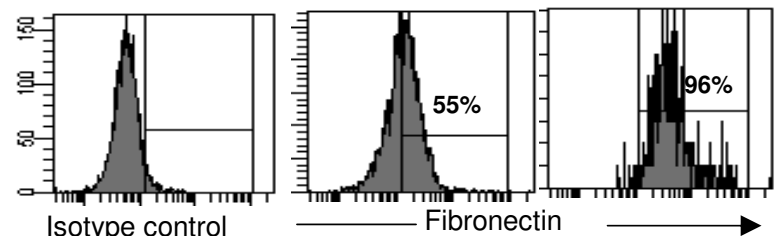

sotype control

Nonactive MM

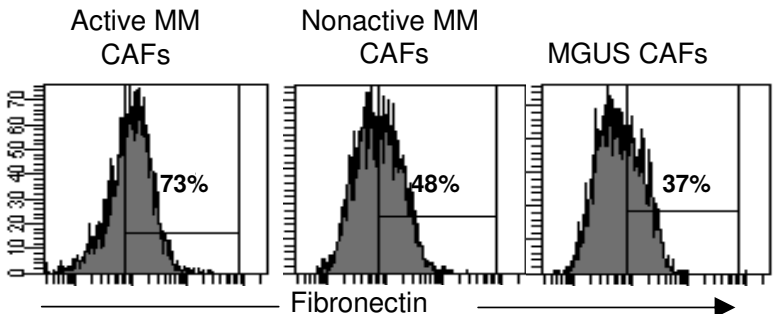

b

C

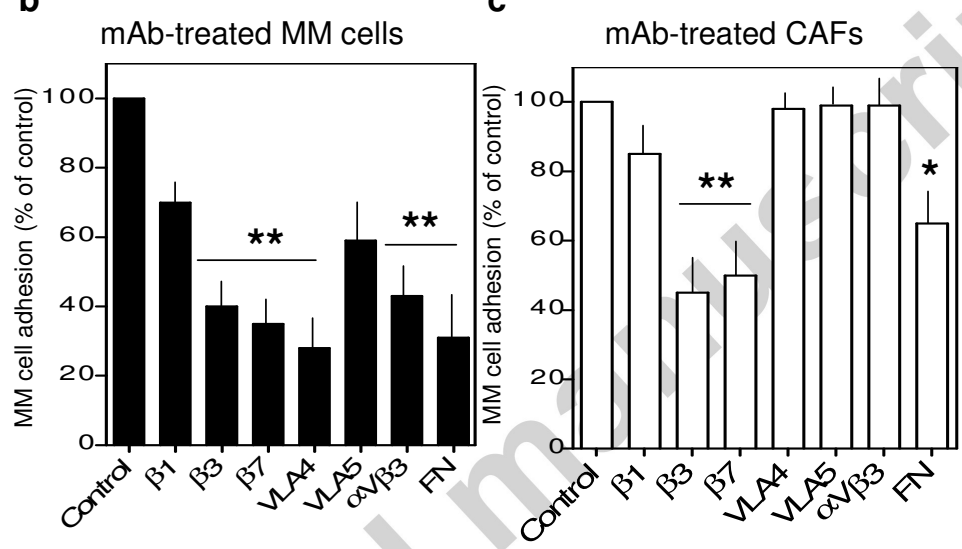

Frassanito et al. Figure 6 

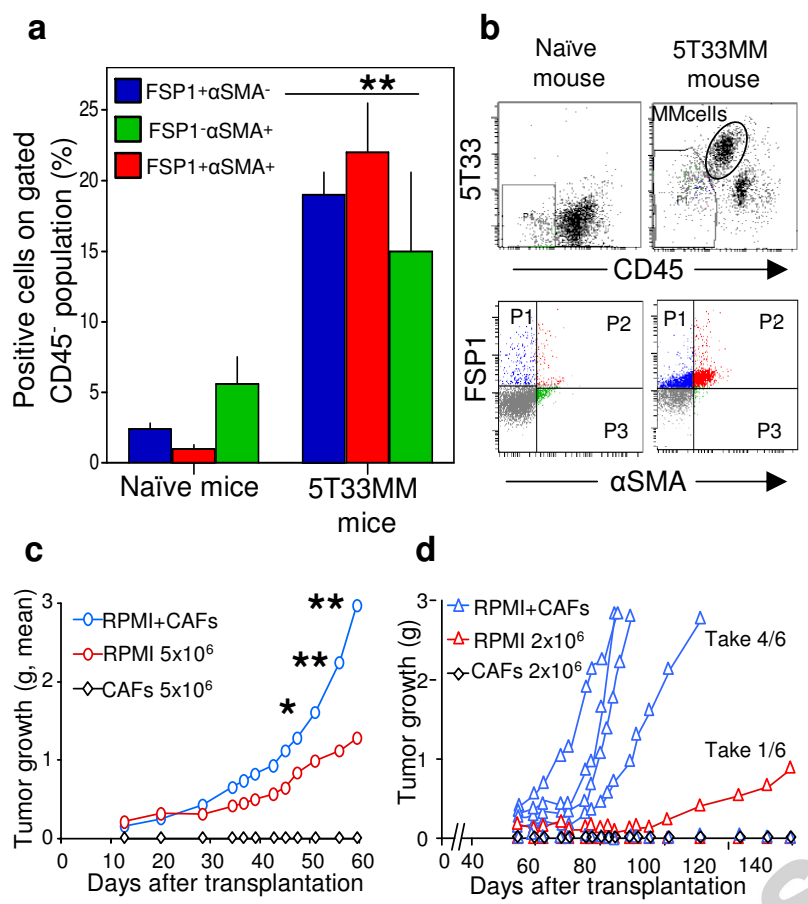

$$
\text { e }
$$

\section{f}

$\mathrm{RPMI}+\mathrm{CAFs}$

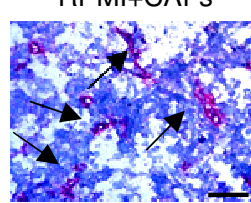

g CD31/DAPI
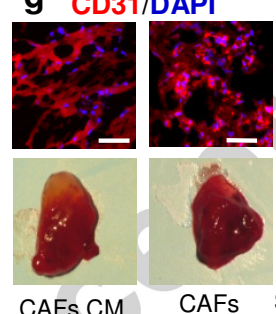

CAFs

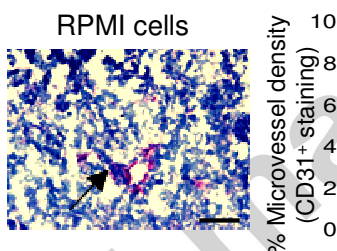

$\begin{array}{ll}\text { RPMI+ } & \text { RPMI } \\ \text { CAFs cells }\end{array}$

CAFs CM

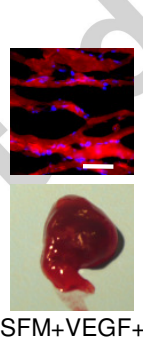

FGF2

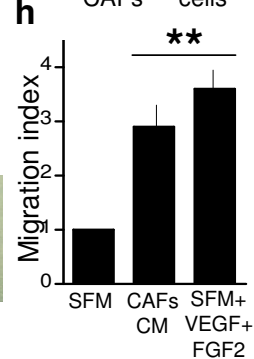

\section{Frassanito et al. Figure 7}



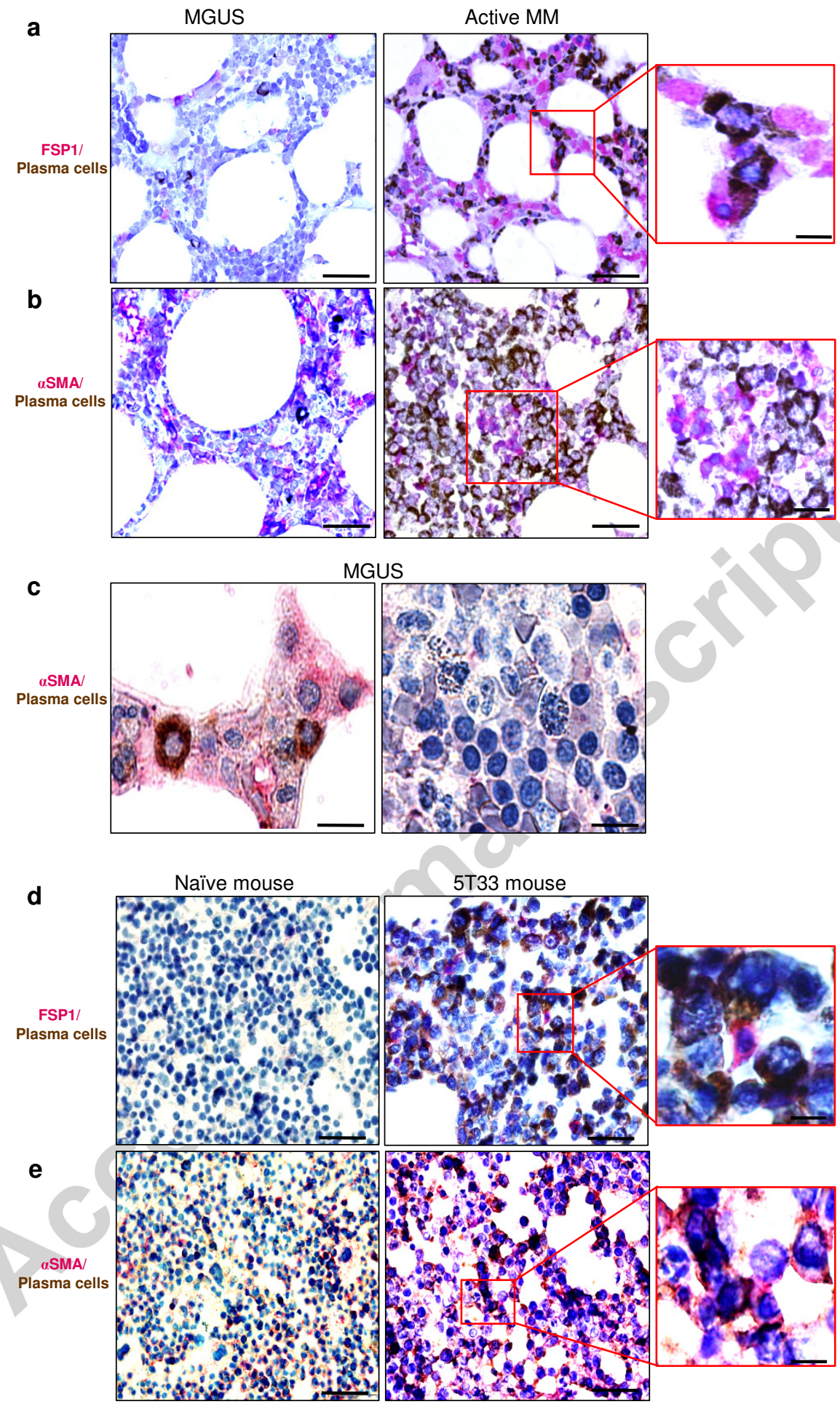

Frassanito et al. Figure 8 